\title{
Decuplet to octet baryon transitions in chiral perturbation theory
}

\author{
Hao-Song Li ${ }^{1,2, a}$, Zhan-Wei Liu ${ }^{3, b}$, Xiao-Lin Chen ${ }^{4, c}$, Wei-Zhen Deng ${ }^{4, d}$, Shi-Lin Zhu ${ }^{2,5, e}$ \\ ${ }^{1}$ Institute of Modern Physics and School of Physics, Northwest University, Xian 710069, China \\ ${ }^{2}$ School of Physics and State Key Laboratory of Nuclear Physics and Technology, Peking University, Beijing 100871, China \\ ${ }^{3}$ School of Physical Science and Technology, Lanzhou University, Lanzhou 730000, China \\ ${ }^{4}$ School of Physics, Peking University, Beijing 100871, China \\ ${ }^{5}$ Collaborative Innovation Center of Quantum Matter, Beijing 100871, China
}

Received: 25 October 2018 / Accepted: 11 January 2019 / Published online: 28 January 2019

(c) The Author(s) 2019

\begin{abstract}
We have systematically investigated the decuplet (T) to octet (B) baryon $(T \rightarrow B \gamma)$ transition magnetic moments to the next-to-next-to-leading order and electric quadruple moments to the next-to-leading order in the framework of the heavy baryon chiral perturbation theory. Our calculation includes the contributions from both the intermediate decuplet and octet baryon states in the loops. Throughout our calculation, we have adopted the small-scale scheme and have not considered the 1/M recoiling corrections directly. We count the octet and decuplet baryon mass splitting $\delta$, the small momenta $p$, and the pion mass $m_{\phi}$ as the same order small-scale parameter, which was denoted as $\epsilon$. Our results for the transition magnetic moments show reasonably good convergence of the chiral expansion. The analytical expressions may be useful to the chiral extrapolation of the lattice simulations of the decuplet electromagnetic properties.
\end{abstract}

\section{Introduction}

The electromagnetic property of the baryons has been an important topic in both theory and experiment. There have been extensive investigations of the baryon magnetic moments [1-31]. On the other hand, the baryon decuplet to octet electromagnetic transition also probes the inner structure and possible deformation of both decuplet and octet baryons. In the past several decades, there have been many investigations of the transition properties from both theoretical and experimental perspectives [32-34].

\footnotetext{
a e-mail: haosongli@pku.edu.cn

b e-mail: liuzhanwei@1zu.edu.cn

ce-mail: chenxl@pku.edu.cn

de-mail:dwz@pku.edu.cn

e e-mail: zhusl@pku.edu.cn
}

The model-independent analysis of the $T \rightarrow B \gamma$ transition amplitude was first performed in Refs. [35,36]. From the spin-parity selection rule, the $T \rightarrow B \gamma$ transition amplitudes contain the magnetic dipole ( $M 1)$, electric quadrupole $(E 2)$, and Coulumb quadrupole $(C 2)$ contributions. In the quark-model picture, a spin flip of a quark in the s-wave state leads to the $M 1$ type of transition, while any d-wave admixture in the octet or the decuplet wave functions allows for the $E 2$ and $C 2$ transitions. Thus, the E2 to M1 ratio $R_{\mathrm{EM}}$ is a signature of the d-wave components and the deviation of the nucleon from spherical symmetry. One of the first successes of the constituent quark model was the prediction of the $\Delta(1232) \rightarrow N \gamma$ transition magnetic moment [37]. If the d-waves are included, the electromagnetic ratios are nonzero [38]. Since then, more sophisticated quark models have been developed to study the $\Delta(1232) \rightarrow N \gamma$ transition [39-46]. In Ref. [47], the authors studied the magnetic moments of $\Delta(1232) \rightarrow N \gamma$ transition with the relativistic quark models improved by chiral corrections.

Although quark model is rather successful in predicting the internal structure of the baryons, the $\Delta(1232) \rightarrow N \gamma$ transition magnetic moment is generally underestimated by $25 \%$ in the constituent quark model. The decuplet and octet baryons are almost degenerate. Moreover, the decuplet baryon nearly entirely decays into the Goldstone boson and octet. It is essential to consider the Goldstone boson cloud effect.

Chiral perturbation theory (ChPT) [48-50] is a very useful framework to take into account the chiral corrections in hadron physics in the low-energy regime [51]. In Ref. [52], heavy baryon chiral perturbation theory (HBChPT) was developed to describe the dynamics of baryons at low energies. "Small scale expansion"(SSE) power counting scheme was proposed in Refs. [53,54], where the mass splitting between the octet and decuplet baryon is treated to be the same order as the masses of pseudo-Goldstone bosons. In 
Ref. [55], the authors studied the $E 2 / M 1$ mixing ratio of the decay $T \rightarrow B \gamma$ with HBChPT to the next-to-leading order. In Ref. [56], the delta-nucleon transition form factors were calculated to $\mathcal{O}\left(\epsilon^{3}\right)$ in the framework of a chiral effective theory, the $\epsilon$ means the same order small-scale parameter: the octet and decuplet baryon mass splitting $\delta$, the small momenta $p$ and the pion mass $m_{\phi}$. The decuplet to octet baryon electromagnetic transition form factors have also been calculated in the quenched and partially quenched chiral perturbation theory in Ref. [57]. An analysis of the electromagnetic transition current was presented to $\mathcal{O}\left(\epsilon^{3}\right)$ in the framework of the non-relativistic SSE chiral effective field theory in Ref. [58]. In Ref. [59], the authors performed a relativistic chiral effective-field theory calculation of the pion electroproduction off the nucleon reaction in the $\Delta(1232)$ resonance region.

Besides the quark models and ChPT, the $T \rightarrow B \gamma$ transition was also studied with various approaches such as the the cloudy bag model [60-62], Skyrme model [63-65], QCD sum rules [66,67], large $N_{C}$ limit $[68,69]$ and lattice QCD [70-76]. Especially, the electromagnetic transition moments of the baryon octet to decuplet were first calculated with quenched lattice QCD simulation in Ref. [70]. The electromagnetic form factors of the $\Delta(1232) \rightarrow N \gamma$ transition were evaluated both in quenched lattice QCD and using two dynamical Wilson fermions in Refs. [71,72]. In Ref. [74], the authors studied the valence quark contributions to the $\Delta(1232) \rightarrow N \gamma$ transition in the lattice QCD regime in the framework of the covariant spectator formalism.

In this work, we calculate the $T \rightarrow B \gamma$ transition amplitudes up to $\mathcal{O}\left(\epsilon^{4}\right)$ and extract the transition magnetic moments to $\mathcal{O}\left(\epsilon^{3}\right)$ in the framework of HBChPT. In our calculation, we do not include the 1/M corrections directly. The 1/M terms can be absorbed into the general Lagrangian within the non relativistic framework as in Ref. [77]. Thus, when we fit the experiment data at last, the $1 / \mathrm{M}$ corrections are included into our final results indirectly. We count the octet and decuplet baryon mass splitting $\delta$, the small momenta $p$ and the pion mass $m_{\phi}$ as the same order smallscale parameter which was denoted as $\epsilon$. We explicitly consider both the octet and decuplet intermediate states in the loop calculation as the octet and decuplet baryons couple strongly. We use the dimensional regularization and modified minimal subtraction scheme to deal with the divergences from the loop corrections. At last, we discuss the convergence of the chiral expansion of the transition magnetic moments. We also systematically calculate the electro quadrupole moments to next-to-leading order and obtain the E2 to M1 ratio $R_{\mathrm{EM}}$ for the decuplet to octet baryon transitions. We collect the M1 and E2 amplitudes and decay width of the decuplet to octet baryon transitions in the Appendix C.
This paper is organized as follows. In Sect. 2, we discuss the decuplet to octet baryon electromagnetic transition form factors. We introduce the effective chiral Lagrangians of the decuplet baryon in Sect. 3. In Sect. 4, we calculate the decuplet to octet baryon transition magnetic moments order by order. We estimate the low-energy constants and present our numerical results in Sect. 5 and conclude in Sect. 6. We collect some useful formulae and the coefficients of the loop corrections in the Appendix A and B.

\section{Decuplet to octet baryon electromagnetic transition form factors}

When the electromagnetic current is sandwiched between the decuplet and octet baryon states, one can write down the general matrix elements which satisfy the gauge invariance, parity conservation and time reversal invariance [36]:

$<B(p)\left|J_{\mu}\right| T\left(p^{\prime}\right)>=e \bar{u}(p) O_{\rho \mu}\left(p^{\prime}, p\right) u^{\rho}\left(p^{\prime}\right)$,

while for the real photons,

$$
\begin{aligned}
O_{\rho \mu}\left(p^{\prime}, p\right)= & \frac{G_{1}}{2 M_{B}}\left(q_{\rho} \gamma_{\mu}-q \cdot \gamma g_{\rho \mu}\right) \gamma_{5} \\
& +\frac{G_{2}}{4 M_{B}^{2}} \frac{1}{M_{B}+M_{T}}\left(q \cdot P g_{\rho \mu}-q_{\rho} P_{\mu}\right) q \gamma_{5} .
\end{aligned}
$$

where $p$ and $p^{\prime}$ are the momenta of the octet and decuplet baryons. In the above equations, $P=\frac{1}{2}\left(p^{\prime}+p\right)$, $q=p^{\prime}-p, M_{B}$ is the octet-baryon mass, $M_{T}$ is the decupletbaryon mass, and $u_{\rho}(p)$ is the Rarita-Schwinger spinor for an on-shell heavy baryon satisfying $p^{\rho} u_{\rho}(p)=0$ and $\gamma^{\rho} u_{\rho}(p)=0$.

In the heavy baryon limit, the baryon field $B$ can be decomposed into the large component $\mathcal{N}$ and the small component $\mathcal{P}$.

$$
\begin{aligned}
B & =e^{-i M_{B} v \cdot x}(\mathcal{N}+\mathcal{P}), \\
\mathcal{N} & =e^{i M_{B} v \cdot x} \frac{1+\psi}{2} B, \mathcal{P}=e^{i M_{B} v \cdot x} \frac{1-\psi}{2} B,
\end{aligned}
$$

where $v_{\mu}=(1, \mathbf{0})$ is the velocity of the baryon. For the decuplet baryon, the large component is denoted as $\mathcal{T}_{\mu}$. Now the decuplet to octet matrix elements of the electromagnetic current $J_{\mu}$ can be parameterized as

$$
<\mathcal{N}(p)\left|J_{\mu}\right| \mathcal{T}\left(p^{\prime}\right)>=e \bar{u}(p) \mathcal{O}_{\rho \mu}\left(p^{\prime}, p\right) u^{\rho}\left(p^{\prime}\right) .
$$

The tensor $\mathcal{O}_{\rho \mu}$ can be parameterized in terms of two Lorentz invariant form factors.

$$
\begin{aligned}
\mathcal{O}_{\rho \mu}\left(p^{\prime}, p\right)= & \frac{G_{1}}{M_{B}}\left(q_{\rho} S_{\mu}-q \cdot S g_{\rho \mu}\right) \\
& +\frac{G_{2}}{4 M_{B}^{2}}\left(q \cdot v g_{\rho \mu}-q_{\rho} v_{\mu}\right) q \cdot S,
\end{aligned}
$$


where $S_{\mu}$ is the covariant spin-operator.

In the following, we shall use Eq. (6) to define the electro quadrupole (E2) and magnetic-dipole (M1) multipole transtion form factors between the decuplet and octet baryons. The multipole form factors are

$$
\begin{aligned}
G_{M 1} & =\frac{2}{3} G_{1}-\frac{\delta}{6 M_{T}} G_{1}-\frac{\delta}{12 M_{B}} G_{2}, \\
G_{E 2} & =\frac{\delta}{6 M_{T}} G_{1}-\frac{\delta}{12 M_{B}} G_{2},
\end{aligned}
$$

where $\delta=M_{T}-M_{B}$ is the octet and decuplet baryon mass splitting. In the isospin symmetry limit, $\delta=0.294 \pm 0.029$ $\mathrm{GeV}$ [32]. We do not consider the mass difference among different decuplet baryons. The $M 1$ and $E 2$ amplitudes $f_{M 1}$ and $f_{E 2}$ are also widely used [78], and they are directly related to $G_{M 1}$ and $G_{E 2}$,

$$
\begin{aligned}
f_{M 1}= & \frac{e}{2 M_{B}}\left(\frac{|q| M_{T}}{M_{B}}\right)^{\frac{1}{2}} G_{M 1} \\
= & \frac{e}{12 M_{B}}\left(\frac{|q|}{M_{T} M_{B}}\right)^{\frac{1}{2}} \\
& {\left[\left(3 M_{T}+M_{B}\right) G_{1}-\frac{M_{T}\left(M_{T}-M_{B}\right)}{2 M_{B}} G_{2}\right], } \\
f_{E 2}= & -\frac{e}{2 M_{B}}\left(\frac{|q| M_{T}}{M_{B}}\right)^{\frac{1}{2}} \frac{2|q| M_{T}}{M_{T}^{2}-M_{B}^{2}} G_{E 2} \\
= & -\frac{e}{6 M_{B}} \frac{|q|}{M_{T}+M_{B}}\left(\frac{|q| M_{T}}{M_{B}}\right)^{\frac{1}{2}}\left(G_{1}-\frac{M_{T}}{2 M_{B}} G_{2}\right),
\end{aligned}
$$

where $|q|=\delta$ in the rest frame of decuplet baryon, $G_{1}$ and $G_{2}$ are defined in Eq. (6).

The E2 to M1 ratio $R_{\mathrm{EM}}$, decay width $\Gamma(T \rightarrow B \gamma)$ and transition magnetic moment $\mu(T \rightarrow B \gamma)$ are expressed as

$$
\begin{aligned}
R_{\mathrm{EM}}=-\frac{G_{E 2}}{G_{M 1}}, & \\
\Gamma(T \rightarrow B \gamma)= & \frac{\alpha}{16} \frac{\left(M_{T}^{2}-M_{B}^{2}\right)^{3}}{M_{T}^{3} M_{B}^{2}}\left(\left|G_{M 1}\left(q^{2}=0\right)\right|^{2}\right. \\
& \left.+3\left|G_{E 2}\left(q^{2}=0\right)\right|^{2}\right), \\
\mu(T \rightarrow B \gamma)= & \frac{2 M_{T}}{M_{T}+M_{B}} G_{M 1}\left(q^{2}=0\right) \frac{e}{2 M_{B}} .
\end{aligned}
$$

where $\alpha=\frac{e^{2}}{4 \pi}=\frac{1}{137}$ is the electromagnetic fine structure constant.

\section{Chiral Lagrangians}

3.1 The strong interaction chiral Lagrangians

The pseudoscalar meson fields are introduced as follows, $\phi=\left(\begin{array}{ccc}\pi^{0}+\frac{1}{\sqrt{3}} \eta & \sqrt{2} \pi^{+} & \sqrt{2} K^{+} \\ \sqrt{2} \pi^{-} & -\pi^{0}+\frac{1}{\sqrt{3}} \eta & \sqrt{2} K^{0} \\ \sqrt{2} K^{-} & \sqrt{2} \bar{K}^{0} & -\frac{2}{\sqrt{3}} \eta\end{array}\right)$.

In the framework of ChPT, the chiral connection and axial vector field are defined as $[79,80]$,

$\Gamma_{\mu}=\frac{1}{2}\left[u^{\dagger}\left(\partial_{\mu}-i r_{\mu}\right) u+u\left(\partial_{\mu}-i l_{\mu}\right) u^{\dagger}\right]$,

$u_{\mu} \equiv \frac{1}{2} i\left[u^{\dagger}\left(\partial_{\mu}-i r_{\mu}\right) u-u\left(\partial_{\mu}-i l_{\mu}\right) u^{\dagger}\right]$,

where

$u^{2}=U=\exp \left(i \phi / F_{0}\right)$.

$F_{0}$ is the decay constant of the pseudoscalar meson in the chiral limit. The experimental value of the pseudoscalar meson decay constant $F_{\pi} \approx 92.4 \mathrm{MeV}$ while $F_{K} \approx 113 \mathrm{MeV}, F_{\eta} \approx$ $116 \mathrm{MeV}$.

The lowest order $\left(\mathcal{O}\left(p^{2}\right)\right)$ pure meson Lagrangian is

$\mathcal{L}_{\pi \pi}^{(2)}=\frac{F_{0}^{2}}{4} \operatorname{Tr}\left[\nabla_{\mu} U\left(\nabla^{\mu} U\right)^{\dagger}\right]$,

where

$\nabla_{\mu} U=\partial_{\mu} U-i r_{\mu} U+i U l_{\mu}$.

For the electromagnetic interaction,

$r_{\mu}=l_{\mu}=-e Q A_{\mu}, Q=\operatorname{diag}\left(\frac{2}{3},-\frac{1}{3},-\frac{1}{3}\right)$.

The spin- $-\frac{1}{2}$ octet field reads

$B=\left(\begin{array}{ccc}\frac{1}{\sqrt{2}} \Sigma^{0}+\frac{1}{\sqrt{6}} \Lambda & \Sigma^{+} & p \\ \Sigma^{-} & -\frac{1}{\sqrt{2}} \Sigma^{0}+\frac{1}{\sqrt{6}} \Lambda & n \\ \Xi^{-} & \Xi^{0} & -\frac{2}{\sqrt{6}} \Lambda\end{array}\right)$.

For the spin- $\frac{3}{2}$ decuplet field, we adopt the Rarita-Schwinger field $T^{\mu} \equiv T^{\mu a b c}[81]$ :

$T^{111}=\Delta^{++}, T^{112}=\frac{1}{\sqrt{3}} \Delta^{+}, T^{122}=\frac{1}{\sqrt{3}} \Delta^{0}$,

$T^{222}=\Delta^{-}, T^{113}=\frac{1}{\sqrt{3}} \Sigma^{*+}$,

$T^{123}=\frac{1}{\sqrt{6}} \Sigma^{* 0}, T^{223}=\frac{1}{\sqrt{3}} \Sigma^{*-}, T^{133}=\frac{1}{\sqrt{3}} \Xi^{* 0}$,

$T^{233}=\frac{1}{\sqrt{3}} \Xi^{*-}, T^{333}=\Omega^{-}$.

The leading order pseudoscalar meson and baryon interaction Lagrangians read $[5,81]$ 


$$
\begin{aligned}
\hat{\mathcal{L}}_{0}^{(1)}= & \operatorname{Tr}\left[\bar{B}\left(i D-M_{B}\right) B\right] \\
& +\operatorname{Tr} \bar{T}^{\mu}\left[-g_{\mu \nu}\left(i D-M_{T}\right)+i\left(\gamma_{\mu} D_{\mu}+\gamma_{\nu} D_{\mu}\right)\right. \\
& \left.-\gamma_{\mu}\left(i D+M_{T}\right) \gamma_{\nu}\right] T^{\nu}, \\
\hat{\mathcal{L}}_{\text {int }}^{(1)}= & \mathcal{C}\left[\operatorname{Tr}\left(\bar{T}^{\mu} u_{\mu} B\right)+\operatorname{Tr}\left(\bar{B} u_{\mu} T^{\mu}\right)\right] \\
& +\mathcal{H} \operatorname{Tr}\left(\bar{T}^{\mu} g_{\mu \nu} \psi_{\nu} \gamma_{5} T^{\nu}\right),
\end{aligned}
$$

where $M_{B}$ is octet-baryon mass, $M_{T}$ is decuplet-baryon mass,

$$
\begin{aligned}
D_{\mu} B= & \partial_{\mu} B+\left[\Gamma_{\mu}, B\right], \\
D^{v}\left(T^{\mu}\right)_{a b c}= & \partial^{v}\left(T^{\mu}\right)_{a b c}+\left(\Gamma^{\nu}\right)_{a}^{d}\left(T^{\mu}\right)_{d b c} \\
& +\left(\Gamma^{\nu}\right)_{b}^{d}\left(T^{\mu}\right)_{a d c}+\left(\Gamma^{\nu}\right)_{c}^{d}\left(T^{\mu}\right)_{a b d} .
\end{aligned}
$$

We also need the second order pseudoscalar meson and decuplet-octet baryon interaction Lagrangians. Recall that

$8 \otimes 8=1 \oplus 8_{1} \oplus 8_{2} \oplus 10 \oplus \overline{10} \oplus 27$,

$8 \otimes 10=8 \oplus 10 \oplus 27 \oplus 35$.

Both $u_{\mu}$ and $u_{\nu}$ transform as the adjoint representation. When the product $u_{\mu} u_{v}$ belongs to the $8_{1}, 8_{2}, \overline{10}$ and 27 flavor representation, we can write down four independent interaction terms of the second order pseudoscalar meson and baryon Lagrangians:

$$
\begin{aligned}
\hat{\mathcal{L}}_{\text {int }}^{(2)}= & \frac{g_{t 1}}{4 M_{B}} \operatorname{Tr}\left(\bar{B}_{b}^{k}\left[u_{\mu}, u_{v}\right]_{a}^{i} \epsilon^{j a b} \gamma^{v} \gamma_{5} T_{i j k}^{\mu}\right) \\
& +\frac{g_{t 2}}{4 M_{B}} \operatorname{Tr}\left(\bar{B}_{b}^{k}\left(u_{\mu} u_{\nu}\right)_{a l}^{i j} \epsilon^{a b l} \gamma^{v} \gamma_{5} T_{i j k}^{\mu}\right) \\
& +\frac{g_{t 4}}{4 M_{B}} \operatorname{Tr}\left(\bar{B}_{b}^{k}\left\{u_{v}, u_{\mu}\right\}_{a}^{i} \epsilon^{j a b} \gamma^{v} \gamma_{5} T_{i j k}^{\mu}\right) \\
& +\frac{g_{t 3}}{4 M_{B}} \operatorname{Tr}\left(\bar{B}_{b}^{l}\left(u_{\mu} u_{v}\right)_{a l}^{i k} \epsilon^{j a b} \gamma^{v} \gamma_{5} T_{i j k}^{\mu}\right)+\text { H.c., }
\end{aligned}
$$

where the superscript denotes the chiral order and $g_{t 1, t 2, t 3, t 4}$ are the coupling constants.

In the framework of HBChPT, the baryon field $B$ is decomposed into the large component $\mathcal{N}$ and the small component $\mathcal{P}$. We denote the large component of the decuplet baryon as $\mathcal{T}_{\mu}$. The leading order nonrelativistic pseudoscalar meson and baryon Lagrangians read [5]

$\mathcal{L}_{0}^{(1)}=\operatorname{Tr}[\overline{\mathcal{N}}(i v \cdot D) \mathcal{N}]-i \overline{\mathcal{T}}^{\mu}(v \cdot D-\delta) \mathcal{T}_{\mu}$,

$\mathcal{L}_{\text {int }}^{(1)}=\mathcal{C}\left(\overline{\mathcal{T}}^{\mu} u_{\mu} \mathcal{N}+\overline{\mathcal{N}} u_{\mu} \mathcal{T}^{\mu}\right)+2 \mathcal{H} \overline{\mathcal{T}}^{\mu} S^{\nu} u_{\nu} \mathcal{T}_{\mu}$,

where $\mathcal{L}_{0}^{(1)}$ and $\mathcal{L}_{\text {int }}^{(1)}$ are the free and interaction parts respectively. The $\phi \mathcal{N} \mathcal{T}$ coupling $\mathcal{C}=-1.2 \pm 0.1$ while the $\phi \mathcal{T} \mathcal{T}$ coupling $\mathcal{H}=-2.2 \pm 0.6$ [82]. We also need the octet baryon axial coupling constants $F$ and $D$.

$\mathcal{L}_{\text {oct }}^{(1)}=2 D \operatorname{Tr}\left(\overline{\mathcal{N}} S^{\mu}\left\{u_{\mu}, \mathcal{N}\right\}\right)+2 F \operatorname{Tr}\left(\overline{\mathcal{N}} S^{\mu}\left[u_{\mu}, \mathcal{N}\right]\right)$

We use $F=0.50 \pm 0.05$ and $D=0.75 \pm 0.08$ [8]. For the pseudoscalar mesons masses, we use $m_{\pi}=0.140 \mathrm{GeV}$, $m_{K}=0.494 \mathrm{GeV}$, and $m_{\eta}=0.550 \mathrm{GeV}$. We use the averaged masses for the octet and decuplet baryons, and $M_{B}=1.158 \mathrm{GeV}, M_{T}=1.452 \mathrm{GeV}$.

The second order pseudoscalar meson and baryon nonrelativistic Lagrangians read

$$
\begin{aligned}
\hat{\mathcal{L}}_{\text {int }}^{(2)}= & \frac{g_{t 1}}{2 M_{B}} \operatorname{Tr}\left(\overline{\mathcal{N}}_{b}^{k}\left[u_{\mu}, u_{\nu}\right]_{a}^{i} \epsilon^{j a b} S^{\nu} \mathcal{T}_{i j k}^{\mu}\right) \\
& +\frac{g_{t 2}}{2 M_{B}} \operatorname{Tr}\left(\overline{\mathcal{N}}_{b}^{k}\left(u_{\mu} u_{v}\right)_{a l}^{i j} \epsilon^{a b l} S^{\nu} \mathcal{T}_{i j k}^{\mu}\right) \\
& +\frac{g_{t 3}}{2 M_{B}} \operatorname{Tr}\left(\overline{\mathcal{N}}_{b}^{k}\left\{u_{\mu}, u_{\nu}\right\}_{a}^{i} \epsilon^{j a b} S^{\nu} \mathcal{T}_{i j k}^{\mu}\right) \\
& +\frac{g_{t 4}}{2 M_{B}} \operatorname{Tr}\left(\overline{\mathcal{N}}_{b}^{l}\left(u_{\mu} u_{\nu}\right)_{a l}^{i k} \epsilon^{j a b} S^{\nu} \mathcal{T}_{i j k}^{\mu}\right)+\text { H.c., }
\end{aligned}
$$

The above Lagrangians contribute to the decuplet to octet baryon transition magnetic moments in diagram (d) of Fig. 2. After loop integration, the contribution of the $g_{t 3}$ term vanishes. Moreover, the contribution of the $g_{t 4}$ term is exactly proportional to that of the $g_{t 2}$ term up to this order. Thus, there are only two linearly independent low energy constants (LECs) $g_{t 1}$ and $g_{t 2}$ which contribute to the present investigations of the decuplet to octet baryon transition form factors up to $\mathcal{O}\left(p^{4}\right)$. Thus in the following, the second order nonrelativistic pseudoscalar meson and baryon Lagrangians will be given in terms of $\tilde{g}_{t 1}=g_{t 1}$ and $\tilde{g}_{t 2}=g_{t 2}+g_{t 4} / 2$ :

$$
\begin{aligned}
\tilde{\mathcal{L}}_{\text {int }}^{(2)}= & \frac{\tilde{g}_{t 1}}{2 M_{B}} \operatorname{Tr}\left(\overline{\mathcal{N}}_{b}^{k}\left[u_{\mu}, u_{\nu}\right]_{a}^{i} \epsilon^{j a b} S^{\nu} \mathcal{T}_{i j k}^{\mu}\right) \\
& +\frac{\tilde{g}_{t 2}}{2 M_{B}} \operatorname{Tr}\left(\overline{\mathcal{N}}_{b}^{k}\left(u_{\mu} u_{\nu}\right)_{a l}^{i j} \epsilon^{a b l} S^{\nu} \mathcal{T}_{i j k}^{\mu}\right)+\text { H.c., }
\end{aligned}
$$

where $\tilde{g}_{t 1}=g_{t 1}$ and $\tilde{g}_{t 2}=g_{t 2}+\frac{1}{2} g_{t 4}$ are the $\phi \phi \mathcal{T} \mathcal{N}$ coupling constants to be fitted. The situation is similar to the decuplet-decuplet case. There are two flavor singlets but only one independent term contributing to the electromagnetic form factors of the decuplet baryons. Only the independent term is listed in Eq. (28) of Ref. [83].

\subsection{The electromagnetic chiral Lagrangians at $\mathcal{O}\left(p^{2}\right)$}

The lowest order $\mathcal{O}\left(p^{2}\right)$ Lagrangian contributes to the magnetic moments of the decuplet baryons at the tree level [5]

$\mathcal{L}_{\mu \mathcal{T}}^{(2)}=\frac{-i b}{2 M_{B}} \operatorname{Tr} \overline{\mathcal{T}}^{\mu} F_{\mu \nu}^{+} \mathcal{T}^{\nu}$,

where the coefficient $b=6.8 \pm 0.4$ was extracted in the calculation of the magnetic moments of the decuplet baryons in Ref. [83]. The chirally covariant QED field strength tensor $F_{\mu \nu}^{ \pm}$is defined as

$$
\begin{aligned}
& F_{\mu \nu}^{ \pm}=u^{\dagger} F_{\mu \nu}^{R} u \pm u F_{\mu \nu}^{L} u^{\dagger}, \\
& F_{\mu \nu}^{R}=\partial_{\mu} r_{\nu}-\partial_{\nu} r_{\mu}-i\left[r_{\mu}, r_{\nu}\right], \\
& F_{\mu \nu}^{L}=\partial_{\mu} l_{\nu}-\partial_{\nu} l_{\mu}-i\left[l_{\mu}, l_{\nu}\right],
\end{aligned}
$$


where $r_{\mu}=l_{\mu}=-e Q A_{\mu}$. The operator $F_{\mu \nu}^{ \pm}$transforms as the adjoint representation. Recall that the direct product $10 \otimes$ $\overline{10}=1 \oplus 8 \oplus 27 \oplus 64$ contains only one adjoint representation. Therefore, there is only one independent interaction term in the $\mathcal{O}\left(p^{2}\right)$ Lagrangians for the magnetic moments of the decuplet baryons.

The lowest order Lagrangians which contribute to the magnetic moments of the octet baryons at the tree level are,

$$
\begin{aligned}
\mathcal{L}_{\mu_{\mathcal{N}}}^{(2)}= & b_{F}^{6} \frac{-i}{4 M_{B}} \operatorname{Tr} \overline{\mathcal{N}}\left[S^{\mu}, S^{\nu}\right]\left[F_{\mu \nu}^{+}, \mathcal{N}\right] \\
& +b_{D}^{6} \frac{-i}{4 M_{B}} \operatorname{Tr} \overline{\mathcal{N}}\left[S^{\mu}, S^{\nu}\right]\left\{F_{\mu \nu}^{+}, \mathcal{N}\right\},
\end{aligned}
$$

where the two LECs were extracted in the calculation of the magnetic moments of the octet baryons in Ref. [8]: $b_{D}^{6}=$ $3.9 \pm 0.4, b_{F}^{6}=3.0 \pm 0.3$.

The lowest order Lagrangians which contribute to the decuplet-octet transition magnetic moments at the tree level are

$$
\begin{aligned}
\mathcal{L}_{\mu_{\mathcal{T} \mathcal{N}}}^{(2)}= & b_{2} \frac{-i}{2 M_{B}} \operatorname{Tr} \overline{\mathcal{T}}^{\mu} F_{\mu \nu}^{+} S^{\nu} \mathcal{N} \\
& +b_{3} \frac{-i}{2 M_{B}} \operatorname{Tr} \overline{\mathcal{T}}^{\mu} F_{\mu \nu}^{+} D^{\nu} \mathcal{N}+\text { H.c. },
\end{aligned}
$$

the $b_{3}$ term does not contribute to the transition magnetic moments.

\subsection{The higher order electromagnetic chiral Lagrangians}

The $\mathcal{O}\left(p^{3}\right)$ Lagrangian which contributes to the electro quadrupole moments at the tree level reads

$$
\mathcal{L}_{\mathbb{Q}_{\mathcal{T} \mathcal{N}}}^{(3)}=c \frac{-1}{4 M_{B}^{2}} \operatorname{Tr}\left(\overline{\mathcal{N}} v^{\mu} \partial_{\nu} F_{\rho \mu}^{+} S^{\nu} \mathcal{T}^{\rho}\right)+\text { H.c.. }
$$

To calculate the transition amplitudes to $\mathcal{O}\left(p^{4}\right)$ and magnetic moments to $\mathcal{O}\left(p^{3}\right)$, we also need the $\mathcal{O}\left(p^{4}\right)$ electromagnetic chiral Lagrangians at the tree level. Recalling Eqs. (26), (27), both $F_{\mu \nu}^{ \pm}$and $\chi^{+}$transform as the adjoint representation. When the product $F_{\mu \nu}^{+} \chi^{+}$belongs to the $8_{1}, 8_{2}, \overline{10}$ and 27 flavor representation, we can write down the chirally invariant $\mathcal{O}\left(p^{4}\right)$ electromagnetic Lagrangians. Therefore, there exist four independent interaction terms in the $\mathcal{O}\left(p^{4}\right)$ chiral Lagrangians. However, for the $\mathcal{O}\left(p^{4}\right)$ LEC contribution, we only need the leading-order terms of the fields $F_{\mu \nu}^{+}$and $\chi^{+}$ which are diagonal matrices. Now, only three independent terms contribute,

$$
\begin{aligned}
\mathcal{L}_{\mu \mathcal{T N}}^{(4)}= & \hat{d}_{1} \frac{-i}{2 M_{B}} \operatorname{Tr}\left(\overline{\mathcal{T}}_{i j k}^{\mu}\left(F_{\mu \nu}^{+} \chi^{+}\right)_{a}^{i} S^{\nu} \epsilon^{j a b} \mathcal{N}_{b}^{k}\right) \\
& +\hat{d}_{2} \frac{-i}{2 M_{B}} \operatorname{Tr}\left(\overline{\mathcal{T}}_{i j k}^{\mu}\left(\epsilon^{a b l}\left(F_{\mu \nu}^{+} \chi^{+}\right)_{a l}^{i j}\right) S^{\nu} \mathcal{N}_{b}^{k}\right) \\
& +\hat{d}_{3} \frac{-i}{2 M_{B}} \operatorname{Tr}\left(\overline{\mathcal{T}}_{i j k}^{\mu}\left(F_{\mu \nu}^{+} \chi^{+}\right)_{a l}^{i k} S^{\nu} \epsilon^{j a b} \mathcal{N}_{b}^{l}\right)+\text { H.c.. }
\end{aligned}
$$

where $\chi^{+}=\operatorname{diag}(0,0,1)$ at the leading order and the factor $m_{s}$ has been absorbed in the LECs $\tilde{d}_{1,2,3}$.

There is one more term which contributes to the transition magnetic moments,

${\mathcal{\mathcal { L } ^ { \prime }}}_{\mu_{\mathcal{T} \mathcal{N}}}^{(4)}=\tilde{b}^{\prime} \frac{-i}{2 M_{B}} \operatorname{Tr}\left(\overline{\mathcal{T}}^{\mu} F_{\mu \nu}^{+} S^{\nu} \mathcal{N}\right) \operatorname{Tr}\left(\chi^{+}\right)+$H.c..

However, its contribution can be absorbed through the renomalization of the LEC $b_{2}$, i.e.

$b_{2} \rightarrow b_{2}+\operatorname{Tr}\left(\chi^{+}\right) \tilde{b}^{\prime}$.

\section{Formalism up to one-loop level}

We apply the standard power counting scheme of HBChPT. The chiral order $D_{\chi}$ of a given diagram is given by [84]

$D_{\chi}=4 N_{L}-2 I_{M}-I_{B}+\sum_{n} n N_{n}$,

where $N_{L}$ is the number of loops, $I_{M}$ is the number of internal pseudoscalar meson lines, $I_{B}$ is the number of internal octet or decuplet baryon lines and $N_{n}$ is the number of the vertices from the $n$th order Lagrangians. As an example, we consider the one-loop diagram (a) in Fig. 2. First of all, the number of the independent loops $N_{L}=1$, the number of the internal pseudoscalar meson lines $I_{M}=2$, the number of the internal octet or decuplet baryon lines $I_{B}=1$. For $N_{1}=2$, and $N_{2}=1$ we obtain $D_{\chi}=4-4-1+2+2=3$.

We use Eq. (44) to count the chiral order $D_{\chi}$ of the matrix element of the current, $e \mathcal{O}_{\rho \mu}$. We count the unit charge $e$ as $\mathcal{O}\left(p^{1}\right)$. The chiral orders of $G_{1}$ and $G_{2}$ are $\left(D_{\chi}-2\right)$ and $\left(D_{\chi}-3\right)$, respectively, since

$e \mathcal{O}_{\rho \mu} \sim e p^{1} G_{1}+e p^{2} G_{2}$.

The chiral order of magnetic dipole $G_{M 1}$ and electric quadrupole $G_{E 2}$ transition moments are $\left(D_{\chi}-1\right)$ and $\left(D_{\chi}-2\right)$ based on Eqs. (7) and (8).

Throughout this work, we assume the exact strong isospin symmetry with $m_{u}=m_{d}$. The tree-level Lagrangians in Eqs. (39), (41) contribute to the decuplet magnetic moments at $\mathcal{O}\left(p^{1}\right)$ and $\mathcal{O}\left(p^{3}\right)$ as shown in Fig. 1. The Clebsch-Gordan coefficients for the various decuplet states are collected in Table 3. All decuplet magnetic moments are given in terms of $\tilde{b}_{2}, \tilde{c}, \tilde{d}_{1,2,3}$, while $\tilde{b}_{2}=\left(\frac{2}{3}-\frac{\delta}{6 M_{T}}\right) b_{2}, \tilde{c}=\frac{\delta}{6 M_{T}} b_{2}-\frac{\delta}{12 M_{B}} c$, $\tilde{d}_{1,2,3}=\left(\frac{2}{3}-\frac{\delta}{6 M_{T}}\right) \hat{d}_{1,2,3}$.

There exist several interesting relations where we use the baryon "B" to denote " $T \rightarrow B \gamma$ " in the last line,

$G_{M 1 \Delta^{+} \rightarrow p \gamma}^{\text {tree }}=G_{M 1 \Delta^{0} \rightarrow n \gamma}^{\text {tree }}$

$G_{M 1 \Sigma^{*-} \rightarrow \Sigma^{-} \gamma}^{\text {tree }}=G_{M 1 \Xi^{*-} \rightarrow \Xi^{-}}^{\text {tree }}$ 


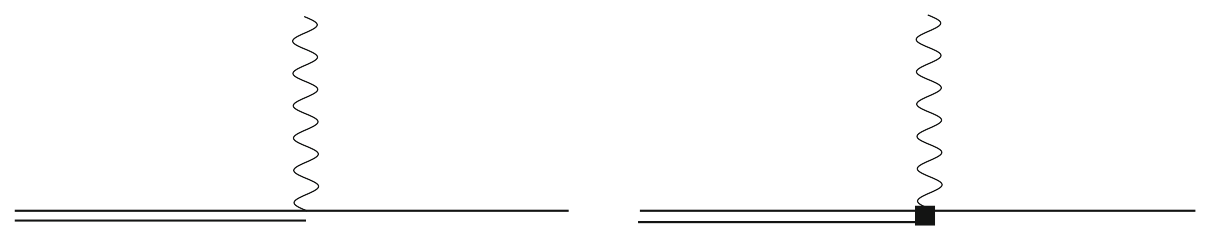

Fig. 1 The $\mathcal{O}\left(p^{2}\right)$ and $\mathcal{O}\left(p^{4}\right)$ tree level diagram where the decuplet (octet) baryon is denoted by the double (single) solid line. The left dot and the right black square represent second- and fourth-order couplings respectively



(a)

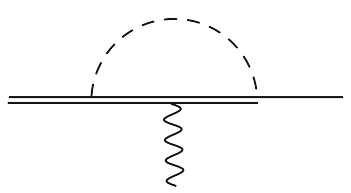

(e)

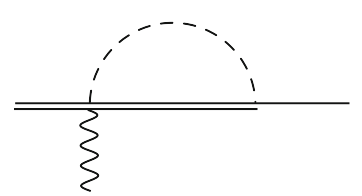

(i)



(m)

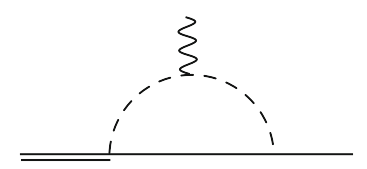

(b)

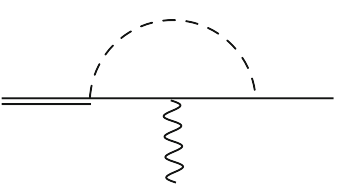

(f)

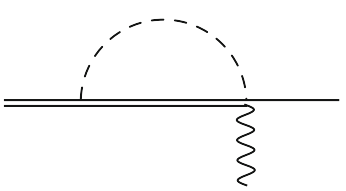

(j)

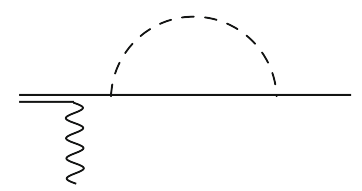

(n)

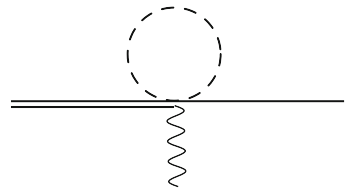

(c)

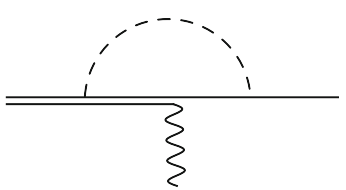

(g)

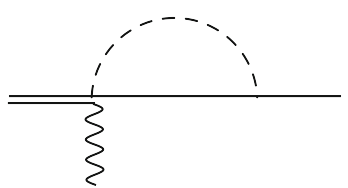

(k)

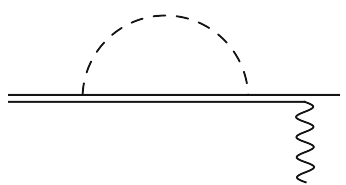

(o)

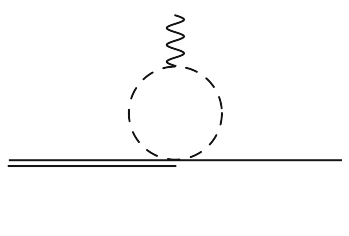

(d)

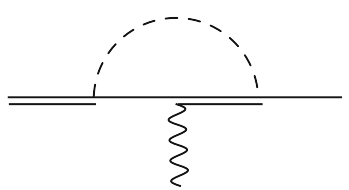

(h)

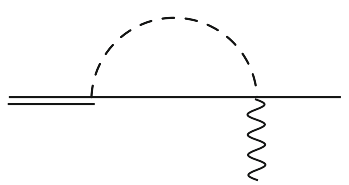

(I)

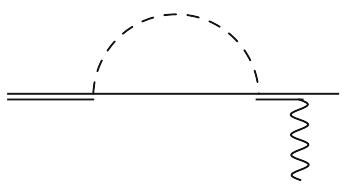

(p)

Fig. 2 The one-loop diagrams where the decuplet (octet) baryon is denoted by the double (single) solid line. The dashed and wiggly lines represent the pseudoscalar meson and photon respectively

$$
\begin{aligned}
& 2 G_{M 1 \Sigma^{* 0} \rightarrow \Sigma^{0} \gamma}^{\text {tree }}+G_{M 1 \Sigma^{*+} \rightarrow \Sigma^{+} \gamma}^{\text {tree }}=G_{M 1 \Sigma^{*-} \rightarrow \Sigma^{-} \gamma}^{\text {tree }} \\
& G_{M 1 \Sigma^{0}}^{\text {tree }}+\sqrt{3} G_{M 1 \Xi^{0}}^{\text {tree }}=G_{M 1 p}^{\text {tree }} \\
& \quad+(\sqrt{3}-1) G_{M 1 \Sigma^{+}}^{\text {tree }}+\sqrt{3} G_{M 1 \Lambda}^{\text {tree }} .
\end{aligned}
$$

There are sixteen Feynman diagrams at one-loop level as shown in Fig. 2. All the vertices in these diagrams come from Eqs. (18), (30-39). In diagrams (a, b), the meson vertex is from the strong interaction terms while the photon vertex is from the meson photon interaction term in Eq. (18). In diagram (c), the photon-meson-baryon vertex is from the $\mathcal{O}\left(p^{2}\right)$ tree level transition magnetic moment interaction in Eq. (39). In diagram (d), the meson-baryon vertex is from the second order pseudoscalar meson and baryon Lagrangian in Eq. (33) while the photon vertex is also from the meson photon interaction term. In diagrams $(\mathrm{e}-\mathrm{h})$, the meson vertex is from the strong interaction terms in Eq. (31) while the photon vertex from the $\mathcal{O}\left(p^{2}\right)$ tree level magnetic moment interaction in Eqs. (35), (38), (39). In diagrams (i-1), the two vertices are from the strong interaction and seagull terms respectively. In diagrams $(m-p)$, the meson vertex is from the strong interaction terms while the photon vertex from the $\mathcal{O}\left(p^{2}\right)$ tree level transition magnetic moment interaction in Eq. (39).

The diagrams (a, b) contribute to the tensor $e \mathcal{O}_{\rho \mu}$ at $\mathcal{O}\left(p^{3}\right)$ while the diagrams $(\mathrm{c}-\mathrm{p})$ contribute at $\mathcal{O}\left(p^{4}\right)$. The diagrams (i-1) vanish in the heavy baryon mass limit. In particular, 
$J_{i} \propto \bar{u} \int \frac{d^{d} l}{(2 \pi)^{d}} q_{\beta} \frac{-i P^{\beta \rho}}{v \cdot l-\delta+i \epsilon} S^{\mu} \frac{i}{l^{2}-m^{2}+i \epsilon} u^{\rho}$

$\propto v_{\rho} u^{\rho}=0$,

$J_{j} \propto \bar{u} \int \frac{d^{d} l}{(2 \pi)^{d}} g_{\beta \mu} \frac{-i P^{\beta \rho}}{v \cdot l-\delta+i \epsilon} S \cdot l \frac{i}{l^{2}-m^{2}+i \epsilon} u^{\rho}$

$\propto S \cdot v=0$,

$J_{k} \propto \bar{u} \int \frac{d^{d} l}{(2 \pi)^{d}} S \cdot l \frac{i}{v \cdot l+i \epsilon} g_{\rho \mu} \frac{i}{l^{2}-m^{2}+i \epsilon} u^{\rho}$

$\propto S \cdot v=0$,

$J_{l} \propto \bar{u} \int \frac{d^{d} l}{(2 \pi)^{d}} S^{\mu} \frac{i}{v \cdot l+i \epsilon} l^{\rho} \frac{i}{l^{2}-m^{2}+i \epsilon} u^{\rho}$

$\propto v_{\rho} u^{\rho}=0$,

where $P^{\beta \rho}$ is the non-relativistic spin- $\frac{3}{2}$ projection operator. In other words, these diagrams do not contribute to the transition magnetic moment in the leading order of the heavy baryon expansion. The diagrams $(\mathrm{m}-\mathrm{p})$ indicate the corrections from the wave function renormalization.

Summing all the contributions in Fig. 2, the leading and next-to-leading order loop corrections to the decuplet to octet baryon transition magnetic moments can be expressed as

$$
\begin{aligned}
\mu_{\mathcal{T} \mathcal{N}}^{(2, \text { loop })}= & \frac{2 M_{T}}{M_{T}+M_{B}} \frac{e}{2 M_{B}}\left[\left(\frac{2}{3}-\frac{\delta}{6 M_{T}}\right) M_{B}\right. \\
& \sum_{\phi=\pi, K}\left(\frac{\beta_{\mathcal{T}}^{\phi}}{F_{\phi}^{2}} \mathcal{C H} a_{\mathcal{T}}^{M \phi}-\frac{\beta_{\mathcal{N}}^{\phi}}{F_{\phi}^{2}} \mathcal{C} b_{\mathcal{N}}^{M \phi}\right) \\
+ & \left.\frac{M_{B}}{3} \sum_{\phi=\pi, K}\left(\frac{-\beta_{\mathcal{T}}^{\phi}}{3 F_{\phi}^{2}} \mathcal{C H} a_{\mathcal{T}}^{E \phi}+\frac{\beta_{\mathcal{N}}^{\phi}}{F_{\phi}^{2}} \mathcal{C} b_{\mathcal{N}}^{E \phi}\right)\right],
\end{aligned}
$$

$$
\begin{aligned}
\mu_{\mathcal{T} \mathcal{N}}^{(3, \text { loop })}= & \frac{2 M_{T}}{M_{T}+M_{B}} \frac{e}{2 M_{B}}\left(\frac{2}{3}-\frac{\delta}{6 M_{T}}\right) \\
& \times\left[\sum_{\phi=\pi, K}\left(-b_{2} \gamma_{c}^{\phi}+\frac{1}{2} \gamma_{d}^{\phi}\right) \frac{m_{\phi}^{2}}{8 \pi^{2} F_{\phi}^{2}} \ln \frac{m_{\phi}}{\lambda}\right. \\
& +\sum_{\phi=\pi, K, \eta}\left(\frac{5}{9 \delta F_{\phi}^{2}} \mathcal{C} \mathcal{H}_{\mathcal{T}}^{\phi} \gamma_{a \mathcal{T}}^{\phi}+\frac{1}{2 F_{\phi}^{2} \delta} \mathcal{C} f_{\mathcal{N}}^{\phi} \gamma_{a \mathcal{N}}^{\phi}\right. \\
& +\frac{5}{12 F_{\phi}^{2}} \mathcal{H} g_{\mathcal{T} \mathcal{N}}^{\phi} b_{2} \gamma_{a \mathcal{T} \mathcal{N}}^{\phi} \\
& \left.\left.+\frac{1}{8 \delta F_{\phi}^{2}} \mathcal{C}^{2} h_{\mathcal{N}}^{\phi} b_{2} \gamma_{a \mathcal{N} \mathcal{T}}^{\phi}\right)\right] \\
& +\frac{2 M_{T}}{M_{T}+M_{B}}\left(\frac{2}{3}-\frac{\delta}{6 M_{T}}\right) \sum_{\phi=\pi, K, \eta} \\
& \times\left[\frac{\mu_{\mathcal{T} \mathcal{N}}^{(1)}}{F_{\phi}^{2}} \frac{-3}{8} n_{\mathcal{N}}^{\phi} \gamma_{f \mathcal{N} 8}^{\phi}\right. \\
& -\frac{\mu_{\mathcal{T} \mathcal{N}}^{(1)}}{4 F_{\phi}^{2}} \mathcal{C}^{2} m_{\mathcal{N}}^{\phi} \gamma_{f \mathcal{N} 10}^{\phi} \\
& \left.+\frac{\mu_{\mathcal{T} \mathcal{N}}^{(1)}}{2 F_{\phi}^{2}} \frac{5}{12} \mathcal{H}^{2} n_{\mathcal{N}}^{\phi} \gamma_{f \mathcal{T} 10}^{\phi}+\frac{\mu_{\mathcal{T} \mathcal{N}}^{(1)}}{8 F_{\phi}^{2}} o_{\mathcal{T}}^{\phi} \mathcal{C}^{2} \gamma_{f \mathcal{T} 8}^{\phi}\right],
\end{aligned}
$$

$\phi=K, \eta$, 


$$
\begin{aligned}
& a_{\mathcal{T}}^{E \phi}=\frac{1}{1152 \pi^{2} \delta} \begin{cases}-104 \delta^{2}+108 \delta^{2} \ln \left(\frac{m_{\phi}^{2}}{\lambda^{2}}\right)+216 \delta \sqrt{\delta^{2}-m_{\phi}^{2}} \operatorname{arccosh}\left(\frac{\delta}{m_{\phi}}\right)+36 m_{\phi}^{2} \operatorname{arccosh}\left(\frac{\delta}{m_{\phi}}\right)^{2} & \\
+9 \pi^{2} m_{\phi}^{2}+48 m_{\phi}^{2}, \\
-104 \delta^{2}+108 \delta^{2} \ln \left(\frac{m_{\phi}^{2}}{\lambda^{2}}\right)-216 \delta \sqrt{m_{\phi}^{2}-\delta^{2}} \arccos \left(\frac{\delta}{m_{\phi}}\right)-36 m_{\phi}^{2} \arccos \left(\frac{\delta}{m_{\phi}}\right)^{2} \\
+9 \pi^{2} m_{\phi}^{2}+48 m_{\phi}^{2}, & \phi=K, \eta,\end{cases} \\
& b_{\mathcal{N}}^{E \phi}=\frac{1}{1152 \pi^{2} \delta^{2}} \begin{cases}-8 \delta^{3}+48 \pi m_{\phi}^{3}+12 \delta^{3} \ln \left(\frac{m_{\phi}^{2}}{\lambda^{2}}\right)+96 i \pi m_{\phi}^{2} \sqrt{\delta^{2}-m_{\phi}^{2}}-24 i \pi \delta^{2} \sqrt{\delta^{2}-m_{\phi}^{2}} & \\
-24\left(4 m_{\phi}^{2}-\delta^{2}\right) \sqrt{\delta^{2}-m_{\phi}^{2}} \operatorname{arccosh}\left(\frac{\delta}{m_{\phi}}\right)+9 \pi^{2} \delta m_{\phi}^{2}+96 \delta m_{\phi}^{2}-36 \delta m_{\phi}^{2} \arccos \left(-\frac{\delta}{m_{\phi}}\right)^{2}, \quad \phi=\pi, \\
-8 \delta^{3}+48 \pi m_{\phi}^{3}+12 \delta^{3} \ln \left(\frac{m_{\phi}^{2}}{\lambda^{2}}\right)-24 \sqrt{m_{\phi}^{2}-\delta^{2}}\left(4 m_{\phi}^{2}-\delta^{2}\right) \arccos \left(-\frac{\delta}{m_{\phi}}\right) & \\
+9 \pi^{2} \delta m_{\phi}^{2}+96 \delta m_{\phi}^{2}-36 \delta m_{\phi}^{2} \arccos \left(-\frac{\delta}{m_{\phi}}\right)^{2}, & \phi=K, \eta,\end{cases} \\
& e_{\mathcal{T}}^{\phi}=\frac{1}{144 \pi^{2}}\left\{\begin{array}{l}
\left(6 \delta^{3}-9 \delta m_{\phi}^{2}\right) \ln \left(\frac{m_{\phi}^{2}}{\lambda^{2}}\right)+12\left(\delta^{2}-m_{\phi}^{2}\right)^{3 / 2} \operatorname{arccosh}\left(\frac{\delta}{m_{\phi}}\right)-2\left(5 \delta^{3}+3 \pi m_{\phi}^{3}-6 \delta m_{\phi}^{2}\right), \quad \phi=\pi, \\
\left(6 \delta^{3}-9 \delta m_{\phi}^{2}\right) \ln \left(\frac{m_{\phi}^{2}}{\lambda^{2}}\right)+12\left(m_{\phi}^{2}-\delta^{2}\right)^{3 / 2} \arccos \left(\frac{\delta}{m_{\phi}}\right)-2\left(5 \delta^{3}+3 \pi m_{\phi}^{3}-6 \delta m_{\phi}^{2}\right), \quad \phi=K, \eta,
\end{array}\right. \\
& 1\left(6 \delta^{3}-9 \delta m_{\phi}^{2}\right) \ln \left(\frac{m_{\phi}^{2}}{\lambda^{2}}\right)+12\left(\delta^{2}-m_{\phi}^{2}\right)^{3 / 2}\left(\operatorname{arccosh}\left(\frac{\delta}{m_{\phi}}\right)-i \pi\right) \\
& f_{\mathcal{N}}^{\phi}=\frac{1}{144 \pi^{2}} \begin{cases}+2\left(-5 \delta^{3}+3 \pi m_{\phi}^{3}+6 \delta m_{\phi}^{2}\right), & \phi=\pi, \\
\left(6 \delta^{3}-9 \delta m_{\phi}^{2}\right) \ln \left(\frac{m_{\phi}^{2}}{\lambda^{2}}\right)-12\left(m_{\phi}^{2}-\delta^{2}\right)^{3 / 2} \arccos \left(-\frac{\delta}{m_{\phi}}\right)+2\left(-5 \delta^{3}+3 \pi m_{\phi}^{3}+6 \delta m_{\phi}^{2}\right), & \phi=K, \eta,\end{cases} \\
& g_{\mathcal{T} \mathcal{N}}^{\phi}=\frac{m_{\phi}^{2}}{48 \pi^{2}}\left[\ln \left(\frac{m_{\phi}^{2}}{\lambda^{2}}\right)-2\right] \text {, } \\
& h_{\mathcal{N} \mathcal{T}}^{\phi}=\frac{1}{216 \pi^{2}} \begin{cases}-14 \delta^{3}+\left(6 \delta^{3}-9 \delta m_{\phi}^{2}\right) \ln \left(\frac{m_{\phi}^{2}}{\lambda^{2}}\right)-6 i \pi\left(\delta^{2}-m_{\phi}^{2}\right)^{3 / 2} & \\
+12\left(\delta^{2}-m_{\phi}^{2}\right)^{3 / 2} \operatorname{arccosh}\left(\frac{\delta}{m_{\phi}}\right)+18 \delta m_{\phi}^{2}, & \phi=\pi, \\
-14 \delta^{3}+\left(6 \delta^{3}-9 \delta m_{\phi}^{2}\right) \ln \left(\frac{m_{\phi}^{2}}{\lambda^{2}}\right)+6\left(m_{\phi}^{2}-\delta^{2}\right)^{3 / 2} \arccos \left(\frac{\delta}{m_{\phi}}\right) & \\
-6\left(m_{\phi}^{2}-\delta^{2}\right)^{3 / 2} \arccos \left(-\frac{\delta}{m_{\phi}}\right)+18 \delta m_{\phi}^{2}, & \phi=K, \eta,\end{cases} \\
& m_{\mathcal{N}}^{\phi}=\frac{1}{16 \pi^{2}}\left\{\begin{array}{l}
2 \delta^{2}+\left(m_{\phi}^{2}-2 \delta^{2}\right) \ln \left(\frac{m_{\phi}^{2}}{\lambda^{2}}\right)-4 \delta \sqrt{\delta^{2}-m_{\phi}^{2}} \operatorname{arccosh}\left(\frac{\delta}{m_{\phi}}\right), \quad \phi=\pi, \\
2 \delta^{2}+\left(m_{\phi}^{2}-2 \delta^{2}\right) \ln \left(\frac{m_{\phi}^{2}}{\lambda^{2}}\right)+4 \delta \sqrt{m_{\phi}^{2}-\delta^{2}} \arccos \left(\frac{\delta}{m_{\phi}}\right), \quad \phi=K, \eta,
\end{array}\right. \\
& n_{\mathcal{N}}^{\phi}=\frac{m_{\phi}^{2}}{16 \pi^{2}} \ln \left(\frac{m_{\phi}^{2}}{\lambda^{2}}\right) \text {, } \\
& o_{\mathcal{T}}^{\phi}=\frac{1}{16 \pi^{2}} \begin{cases}\left(m_{\phi}^{2}-2 \delta^{2}\right) \ln \left(\frac{m_{\phi}^{2}}{\lambda^{2}}\right)+2 \delta\left(\delta+2 i \pi \sqrt{\delta^{2}-m_{\phi}^{2}}\right)-4 \delta \sqrt{\delta^{2}-m_{\phi}^{2}} \operatorname{arccosh}\left(\frac{\delta}{m_{\phi}}\right), & \phi=\pi, \\
2 \delta^{2}+\left(m_{\phi}^{2}-2 \delta^{2}\right) \ln \left(\frac{m_{\phi}^{2}}{\lambda^{2}}\right)-4 \delta \sqrt{m_{\phi}^{2}-\delta^{2}} \arccos \left(-\frac{\delta}{m_{\phi}}\right), & \phi=K, \eta .\end{cases}
\end{aligned}
$$


where $\lambda=1 \mathrm{GeV}$ is the renormalization scale, $\phi$ denotes different pseudoscalar mesons. In Eqs. (51), (52), the decay constant $F_{\phi}$ reads: $F_{\pi} \approx 92.4 \mathrm{MeV}, F_{K} \approx 113 \mathrm{MeV}, F_{\eta} \approx$ $116 \mathrm{MeV}$. The coefficients $\beta_{\mathcal{T}}^{\phi}$ and $\beta_{\mathcal{N}}^{\phi}$ arise from the decuplet and octet intermediate states respectively. We use the number $n$ within the parenthesis in the superscript of $X^{(n, \ldots)}$ to indicate the chiral order of $X . \gamma_{c}^{\phi}, \gamma_{d}^{\phi}, \gamma_{a \mathcal{T}}^{\phi}, \gamma_{a \mathcal{N}}^{\phi}, \gamma_{a \mathcal{T} \mathcal{N}}^{\phi}, \gamma_{a \mathcal{N} \mathcal{T}}^{\phi}$, $\gamma_{f \mathcal{N} 8}^{\phi}, \gamma_{f \mathcal{N} 10}^{\phi}, \gamma_{f \mathcal{T} 10}^{\phi}$ and $\gamma_{f \mathcal{T} 8}^{\phi}$ arise from the diagrams in Fig. 2. We collect their explicit expressions in Tables 7, 8, 9, $10,11,12$ in the Appendix B.

With the low energy counter terms and loop contributions Eqs. $(51,52)$, we obtain the magnetic moments,

$\mu_{\mathcal{T} \mathcal{N}}=\left\{\mu_{\mathcal{T} \mathcal{N}}^{(1)}\right\}+\left\{\mu_{\mathcal{T} \mathcal{N}}^{(2, \text { loop })}\right\}+\left\{\mu_{\mathcal{T} \mathcal{N}}^{(3, \text { tree })}+\mu_{\mathcal{T} \mathcal{N}}^{(3, \text { loop })}\right\}$

where $\mu_{\mathcal{T} \mathcal{N}}^{(1)}$ and $\mu_{\mathcal{T} \mathcal{N}}^{(3, \text { tree })}$ are the tree-level magnetic moments from Eqs. (39), (41).

Summing all the contributions to electric quadrupole moments in Fig. 2, the leading and next-to-leading order loop corrections can be expressed as

$$
\begin{aligned}
G_{E 2}^{(1, \text { loop })}= & \frac{\delta}{6 M_{T}} M_{B} \sum_{\phi=\pi, K}\left(\frac{\beta_{\mathcal{T}}^{\phi}}{F_{\phi}^{2}} \mathcal{C H} a_{\mathcal{T}}^{M \phi}-\frac{\beta_{\mathcal{N}}^{\phi}}{F_{\phi}^{2}} \mathcal{C} b_{\mathcal{N}}^{M \phi}\right) \\
& +\frac{M_{B}}{3} \sum_{\phi=\pi, K}\left(\frac{-\beta_{\mathcal{T}}^{\phi}}{3 F_{\phi}^{2}} \mathcal{C H} a_{\mathcal{T}}^{E \phi}+\frac{\beta_{\mathcal{N}}^{\phi}}{F_{\phi}^{2}} \mathcal{C} b_{\mathcal{N}}^{E \phi}\right)
\end{aligned}
$$

$G_{E 2}^{(2, \text { loop })}=0$

From the tensor $e \mathcal{O}_{\rho \mu \sigma}$ up to $\mathcal{O}\left(p^{4}\right)$, with the low energy counter terms and loop contributions Eqs. (65), (66), we obtain the electric quadrupole moments at the next-to-leading order,

$G_{E 2}=\left\{G_{E 2}^{(1, \text { tree })}+G_{E 2}^{(1, \text { loop })}\right\}+\left\{G_{E 2}^{(2, \text { loop })}\right\}$

where $G_{E 2}^{(1, \text { tree) }}$ is the tree-level electro quadrupole moments from Eq. (40). Thus, one obtain the form factors $G_{1}$ and $G_{2}$ in Eq. (6) when $q^{2}=0$,

$$
\begin{aligned}
G_{1} & =\frac{-3 M_{T}}{2 M_{T}-\delta}\left(G_{E 2}-G_{M 1}\right), \\
G_{2} & =\frac{-6 M_{B}}{\delta\left(-2 M_{T}+\delta\right)}\left[\delta G_{M 1}+G_{E 2}\left(-4 M_{T}+\delta\right)\right],
\end{aligned}
$$

\section{Numerical results and discussions}

Throughout this work, there are 15 parameters: $\delta, \mathcal{C}, \mathcal{H}, D$, $F, b, b_{D}^{6}, b_{F}^{6}, \tilde{c}, b_{2}, \tilde{d}_{1,2,3}, \tilde{g}_{t 1}, \tilde{g}_{t 2}$. The first 9 parameters can be taken from the references, we compile them in Table 1. The error bars of $\mathcal{C}, \mathcal{H}, b$ and $\tilde{c}$ are quoted from the references while the error bars of the other parameters we roughly take for $10 \%$. The last 6 parameters have to be fitted from the experimental inputs, we compile them in Table 2. However, we only have three accurate experimental inputs shown in Table 3. The last 6 parameters are inevitably uncertain, as the data set is not rich enough to fix the LECs indeed.

We collect our numerical results of the baryon decuplet to octet baryon transition magnetic moments to the nextto-next-to-leading order in Table 3. We also compare the numerical results of the transition magnetic moments when the chiral expansion is truncated up to $\mathcal{O}\left(p^{1}\right), \mathcal{O}\left(p^{2}\right)$ and $\mathcal{O}\left(p^{3}\right)$ respectively in Table 4 .

At the leading order $\mathcal{O}\left(p^{1}\right)$, there is only one unknown low energy constant $\tilde{b}_{2}$. We use the precise experimental measurement of the $\Delta \rightarrow N \gamma$ transition magnetic moment $\mu_{\Delta \rightarrow N \gamma}=(-3.43 \pm 0.15) \mu_{N}$ as input to extract $\tilde{b}_{2}=$ $2.97 \pm 0.13$. The magnetic moments of the other decuplet baryons are given in the second column in Table 4 . Notice that the $\mathcal{O}\left(p^{1}\right)$ tree level transitions $\Delta^{+} \rightarrow p \gamma$ and $\Delta^{0} \rightarrow n \gamma$ are the same. In fact, this equation holds to every order because of the exact $\mathrm{SU}(2)$ spin-flavor symmetry. The $\mathcal{O}\left(p^{1}\right)$ tree level transitions $\Sigma^{*-} \rightarrow \Sigma^{-} \gamma$ and $\Xi^{*-} \rightarrow \Xi^{-} \gamma$ are zero because of the famous U-spin symmetry as can be seen from Table 15 in the Appendix D.

Up to $\mathcal{O}\left(p^{2}\right)$, we need include both the leading tree-level magnetic moments and the $\mathcal{O}\left(p^{2}\right)$ loop corrections. At this order, all the coupling constants are well-known. There do not exist new LECs. Again, we use the experimental value of the $\Delta \rightarrow N \gamma$ transition magnetic moment $\mu_{\Delta \rightarrow N \gamma}=(-3.43 \pm$ $0.15) \mu_{N}$ as input to extract the LEC $\tilde{b}_{2}=4.87 \pm 0.13$. We list the numerical results in the third column in Table 4, where the errors in the brackets are dominated by the errors of the coupling constants $\mathcal{C}, \mathcal{H}$ in Eq. (31). Notice that the $\Sigma^{*+} \rightarrow \Sigma^{+} \gamma$ transition magnetic moment to $\mathcal{O}\left(p^{2}\right)$ is quite small compared to the experimental value, which will be improved when the $\mathcal{O}\left(p^{3}\right)$ contribution is included. In other approaches in Table 5, the transition magnetic moment of $\Sigma^{*+} \rightarrow \Sigma^{+} \gamma$ is also smaller than that of $\Delta \rightarrow N \gamma$. Up to $\mathcal{O}\left(p^{2}\right)$, SU(3) symmetry is kept.

To $\mathcal{O}\left(p^{3}\right)$, we introduce the mass of the strange quark in Eq. (41) which breaks the $\mathrm{SU}(3)$ symmetry. Up to $\mathcal{O}\left(p^{3}\right)$, there are six unknown LECs: $\tilde{b}_{2}, \tilde{g}_{t 1, t 2}, \tilde{d}_{1,2,3}$. Two schemes will be introduced to fit all LECs. In the first fit (Fit A), we use the experimental values of the transition magnetic moments of $\Delta \rightarrow N \gamma, \Sigma^{*+} \rightarrow \Sigma^{+} \gamma, \Sigma^{* 0} \rightarrow \Lambda \gamma$, the upper limit of the transition magnetic moment of $\Xi^{* 0} \rightarrow$ $\Xi^{0} \gamma$ and $\mu_{\Sigma^{*-} \rightarrow \Sigma^{-} \gamma}=\mu_{\Xi^{*-} \rightarrow \Xi^{-} \gamma}=(0 \pm 0.85) \mu_{N}$ to extract the six LECs: $\tilde{b}_{2}=5.26 \pm 3.28, \tilde{g}_{t 1}=-3.73 \pm 7.96$, $\tilde{g}_{t 2}=-12.91 \pm 14.3, \tilde{d}_{1}=0.93 \pm 4.02, \tilde{d}_{2}=0.10 \pm 1.34$, $\tilde{d}_{3}=-0.96 \pm 0.62$. We list the numerical results up to $\mathcal{O}\left(p^{3}\right)$ in the fourth column in Table 4. 
Table 1 The 9 parameters taken from the references

\begin{tabular}{llllllllll}
\hline Parameters & $\delta$ & $\mathcal{C}$ & $\mathcal{H}$ & $D$ & $F$ & $b$ & $b_{D}^{6}$ & $b_{F}^{6}$ & $\tilde{c}$ \\
\hline Equation & Eq. (30) & Eq. (31) & Eq. (31) & Eq. (32) & Eq. (32) & Eq. (35) & Eq. (38) & Eq. (38) & Eq. (40) \\
Reference & Ref [32] & Ref [82] & Ref [82] & Ref [82] & Ref [82] & Ref. [83] & Ref. [8] & Ref. [8] & Ref. [32] \\
Value & $0.294 \pm 0.029$ & $-1.2 \pm 0.1$ & $-2.2 \pm 0.6$ & $0.75 \pm 0.08$ & $0.50 \pm 0.05$ & $6.8 \pm 0.4$ & $3.9 \pm 0.4$ & $3.0 \pm 0.3$ & $0.475 \pm 0.143$ \\
\hline
\end{tabular}

Table 2 The 6 parameters to be fitted

\begin{tabular}{lllllll}
\hline Parameters & $\tilde{b}_{2}$ & $\tilde{d}_{1}$ & $\tilde{d}_{2}$ & $\tilde{d}_{3}$ & $\tilde{g}_{t 1}$ & $\tilde{g}_{t 2}$ \\
\hline Relations & $\tilde{b}_{2}=\left(\frac{2}{3}-\frac{\delta}{6 M_{T}}\right) b_{2}$ & $\tilde{d}_{1}=\left(\frac{2}{3}-\frac{\delta}{6 M_{T}}\right) \hat{d}_{1}$ & $\tilde{d}_{2}=\left(\frac{2}{3}-\frac{\delta}{6 M_{T}}\right) \hat{d}_{2}$ & $\tilde{d}_{3}=\left(\frac{2}{3}-\frac{\delta}{6 M_{T}}\right) \hat{d}_{3}$ & $\tilde{g}_{t 1}=g_{t 1}$ & $\tilde{g}_{t 2}=g_{t 2}+\frac{1}{2} g_{t 4}$ \\
Equation & Eq. (39) & Eq. (41) & Eq. $(41)$ & Eq. (41) & Eq. (34) & Eq. (34) \\
Fit A & $5.26 \pm 3.28$ & $0.93 \pm 4.02$ & $0.10 \pm 1.34$ & $-0.96 \pm 0.62$ & $-3.73 \pm 7.96$ & $-12.91 \pm 14.3$ \\
Fit B & $4.22 \pm 0.53$ & $-0.96 \pm 0.72$ & $-0.32 \pm 0.31$ & $-1.62 \pm 0.34$ & $-8.18 \pm 7.55$ & $-31.69 \pm 17.60$ \\
\hline
\end{tabular}

Table 3 The decuplet to octet baryon transition $G_{M 1}\left(q^{2}=0\right)$ to the next-to-next-to-leading order (in unit of 1). Actually, we use the branching ratios in PDG to extract the corresponding transition $\left|G_{M 1}\left(q^{2}=0\right)\right|_{\exp }$

\begin{tabular}{|c|c|c|c|c|c|c|c|}
\hline $\operatorname{Process}\left(G_{\mathrm{M} 1}\right)$ & $\mathcal{O}\left(p^{1}\right)$ & $\mathcal{O}\left(p^{2}\right)$ & $\mathcal{O}\left(p^{3}\right)$ tree & $\mathcal{O}\left(p^{3}\right)$ & Total Fit A & Total Fit B & $\left|G_{M 1}\left(q^{2}=0\right)\right|_{\exp }$ \\
\hline$\Delta^{+} \rightarrow p \gamma$ & $-\frac{2}{\sqrt{3}} \tilde{b}_{2}$ & 1.99 & 0 & $1.83+0.10 \tilde{g}_{t 1}+0.04 \tilde{g}_{t 2}$ & -3.18 & -3.22 & $3.12(14)$ \\
\hline$\Delta^{0} \rightarrow n \gamma$ & $-\frac{2}{\sqrt{3}} \tilde{b}_{2}$ & 1.99 & 0 & $1.83+0.10 \tilde{g}_{t 1}+0.04 \tilde{g}_{t 2}$ & -3.18 & -3.22 & $3.12(14)$ \\
\hline$\Sigma^{*+} \rightarrow \Sigma^{+} \gamma$ & $\frac{2}{\sqrt{3}} \tilde{b}_{2}$ & -3.15 & $\frac{2}{3 \sqrt{3}} \tilde{d}_{1}+\frac{4 \sqrt{3}}{9} \tilde{d}_{2}$ & $-1.46-0.15 \tilde{g}_{t 1}-0.12 \tilde{g}_{t 2}$ & 4.05 & 4.03 & $4.05(49)$ \\
\hline$\Sigma^{* 0} \rightarrow \Sigma^{0} \gamma$ & $-\frac{1}{\sqrt{3}} \tilde{b}_{2}$ & 1.72 & $-\frac{2}{3 \sqrt{3}} \tilde{d}_{1}-\frac{\sqrt{3}}{9} \tilde{d}_{2}$ & $0.72+0.09 \tilde{g}_{t 1}+0.06 \tilde{g}_{t 2}$ & -2.12 & -2.11 & - \\
\hline$\Sigma^{* 0} \rightarrow \Lambda \gamma$ & $\tilde{b}_{2}$ & -1.98 & $\frac{1}{3} \tilde{d}_{2}+\frac{2 \sqrt{3}}{3} \tilde{d}_{3}$ & $-0.49-0.09 \tilde{g}_{t 1}-0.10 \tilde{g}_{t 2}$ & 3.29 & 3.22 & $3.25(46)$ \\
\hline$\Sigma^{*-} \rightarrow \Sigma^{-} \gamma$ & 0 & 0.29 & $-\frac{2}{3 \sqrt{3}} \tilde{d}_{1}+\frac{2 \sqrt{3}}{9} \tilde{d}_{2}$ & $-0.01+0.04 \tilde{g}_{t 1}$ & -0.19 & -0.20 & $<0.78(04)$ \\
\hline$\Xi^{* 0} \rightarrow \Xi^{0} \gamma$ & $\frac{2}{\sqrt{3}} \tilde{b}_{2}$ & -3.15 & $\frac{2}{3 \sqrt{3}} \tilde{d}_{1}+\frac{4 \sqrt{3}}{9} \tilde{d}_{2}+\frac{2 \sqrt{3}}{3} \tilde{d}_{3}$ & $0.49-0.15 \tilde{g}_{t 1}-0.12 \tilde{g}_{t 2}$ & 4.89 & 4.10 & $<4.90(53)$ \\
\hline$\Xi^{*-} \rightarrow \Xi^{-} \gamma$ & 0 & 0.29 & $-\frac{2}{3 \sqrt{3}} \tilde{d}_{1}+\frac{2 \sqrt{3}}{9} \tilde{d}_{2}$ & $0.36+0.04 \tilde{g}_{t 1}$ & 0.18 & 0.18 & $<4.90(53)$ \\
\hline
\end{tabular}

Table 4 The decuplet to octet baryon transition magnetic moments when the chiral expansion is truncated up to $\mathcal{O}\left(p^{1}\right), \mathcal{O}\left(p^{2}\right)$, and $\mathcal{O}\left(p^{3}\right)$, respectively (in unit of $\mu_{N}$ ). Actually, we use the branching ratios in PDG to extract the corresponding transition magnetic moments

\begin{tabular}{|c|c|c|c|c|c|}
\hline $\operatorname{Process}\left(\mu_{\mathcal{T} \mathcal{N}}\right)$ & $\mathcal{O}\left(p^{1}\right)$ & $\mathcal{O}\left(p^{2}\right)$ & $\mathcal{O}\left(p^{3}\right)$ Fit $\mathrm{A}$ & $\mathcal{O}\left(p^{3}\right)$ Fit $\mathrm{B}$ & $\left|\mu_{\mathcal{T} \mathcal{N}}\right|_{\exp }$ \\
\hline$\Delta^{+} \rightarrow p \gamma$ & $-3.43(15)$ & $-3.43(65)$ & $-3.50(95)$ & $-3.54(96)$ & $3.43(15)$ \\
\hline$\Delta^{0} \rightarrow n \gamma$ & $-3.43(15)$ & $-3.43(65)$ & $-3.50(95)$ & $-3.54(96)$ & $3.43(15)$ \\
\hline$\Sigma^{*+} \rightarrow \Sigma^{+} \gamma$ & $3.43(15)$ & $2.16(87)$ & $4.46(1.30)$ & $4.43(1.28)$ & $4.45(54)$ \\
\hline$\Sigma^{* 0} \rightarrow \Sigma^{0} \gamma$ & $-1.72(08)$ & $-0.92(46)$ & $-2.34(69)$ & $-2.32(68)$ & - \\
\hline$\Sigma^{* 0} \rightarrow \Lambda \gamma$ & $2.97(13)$ & $2.69(61)$ & $3.62(92)$ & $3.54(90)$ & $3.69(50)$ \\
\hline$\Sigma^{*-} \rightarrow \Sigma^{-} \gamma$ & 0 & $0.32(06)$ & $-0.21(09)$ & $-0.22(09)$ & $<0.85(05)$ \\
\hline$\Xi^{* 0} \rightarrow \Xi^{0} \gamma$ & $3.43(15)$ & $2.16(87)$ & $5.38(1.39)$ & $4.51(1.16)$ & $<5.39(58)$ \\
\hline$\Xi^{*-} \rightarrow \Xi^{-} \gamma$ & 0 & $0.32(06)$ & $0.20(08)$ & $0.19(08)$ & $<5.39(58)$ \\
\hline
\end{tabular}

In order to study the convergence of the chiral expansion, we show the numerical results at each order for the transition magnetic moments:

$$
\begin{aligned}
\mu_{\Delta^{+} \rightarrow p \gamma} & =-6.68 \times(1-0.33-0.15)=-3.50, \\
\mu_{\Delta^{0} \rightarrow n \gamma} & =-6.68 \times(1-0.33-0.15)=-3.50,
\end{aligned}
$$

$$
\begin{aligned}
\mu_{\Sigma^{*+} \rightarrow \Sigma^{+} \gamma} & =6.68 \times(1-0.52+0.19)=4.46, \\
\mu_{\Sigma^{* 0} \rightarrow \Sigma^{0} \gamma} & =-3.34 \times(1-0.57+0.27)=-2.34, \\
\mu_{\Sigma^{* 0} \rightarrow \Lambda \gamma} & =5.78 \times(1-0.38+0.01)=3.62, \\
\mu_{\Sigma^{*-} \rightarrow \Sigma^{-} \gamma} & =0.32 \times(0+1-1.65)=-0.21, \\
\mu_{\Xi^{* 0} \rightarrow \Xi^{0} \gamma} & =6.68 \times(1-0.52+0.32)=5.38,
\end{aligned}
$$


Table 5 Comparison of the decuplet to octet baryon transition magnetic moments in literature including lattice QCD (LQCD) [70], chiral quark model (ChQM) [85], relativistic quark model (RQM) [86], effective mass quark model (EQM) [87], meson cloud (MS) [88], U-spin [89],
QCD sum rules (QCD-SR) [67,90], large $N_{c}$ [91,92], and PDG [32](in unit of $\mu_{N}$ ). Actually, we use the branching ratios in PDG to extract the corresponding transition magnetic moments

\begin{tabular}{|c|c|c|c|c|c|c|c|}
\hline Process & $\Delta \rightarrow N \gamma$ & $\Sigma^{*+} \rightarrow \Sigma^{+} \gamma$ & $\Sigma^{* 0} \rightarrow \Sigma^{0} \gamma$ & $\Sigma^{* 0} \rightarrow \Lambda \gamma$ & $\Sigma^{*-} \rightarrow \Sigma^{-} \gamma$ & $\Xi^{* 0} \rightarrow \Xi^{0} \gamma$ & $\Xi^{*-} \rightarrow \Xi^{-} \gamma$ \\
\hline LQCD [70] & 2.46 & 2.61 & 1.07 & - & -0.47 & -2.77 & 0.47 \\
\hline ChQM [85] & -3.31 & 2.17 & - & -2.74 & -0.59 & 2.23 & -0.59 \\
\hline RQM [86] & 3.25 & 2.59 & 1.07 & 2.86 & -0.46 & 2.71 & -0.47 \\
\hline EQM [87] & 2.63 & 2.33 & 1.02 & 2.28 & 0.30 & 2.33 & 0.30 \\
\hline MS [88] & 3.32 & 3.54 & 1.61 & 3.39 & -0.34 & 3.62 & -0.42 \\
\hline U-spin [89] & - & 3.22 & 1.61 & 2.68 & 0 & 3.21 & - \\
\hline QCD-SR [67] & 3.86 & 3.38 & 1.47 & 4.44 & -0.57 & -1.24 & 0.23 \\
\hline QCD-SR [90] & -2.76 & 2.24 & 1.01 & -2.46 & -0.22 & 2.46 & -0.27 \\
\hline Large $N c$ [91] & 3.51 & 2.96 & 1.34 & 2.96 & -0.27 & 2.96 & - \\
\hline Large $N c$ [92] & 3.51 & 2.97 & 1.39 & 2.93 & -0.19 & 2.96 & -0.19 \\
\hline This work (fit A) & -3.50 & 4.46 & -2.34 & 3.62 & -0.21 & 5.38 & 0.20 \\
\hline This work (fit B) & -3.54 & 4.43 & -2.34 & 3.54 & -0.22 & 4.51 & 0.19 \\
\hline$\left|G_{M 1}\left(q^{2}=0\right)\right|_{\exp } / \mu_{N}$ & $3.43(15)$ & $4.45(54)$ & - & $3.69(50)$ & $<0.85(05)$ & $<5.39(58)$ & $<5.39(58)$ \\
\hline
\end{tabular}

$\mu_{\Xi^{*-} \rightarrow \Xi^{-} \gamma}=0.32 \times(0+1-0.38)=0.20$.

For the U-spin forbidden processes, their magnetic moments vanish at $\mathcal{O}\left(p^{1}\right)$. Their total magnetic moments arise from the loop contributions at $\mathcal{O}\left(p^{2,3}\right)$ and the treelevel LECs $d_{1,2,3}$ at $\mathcal{O}\left(p^{3}\right)$ which are related to the strange quark mass correction. For the other processes, one observes rather good convergence of the chiral expansion and the leading order term dominates in these channels.

In the second fit (Fit B), considering transition magnetic moments of $\Sigma^{*+} \rightarrow \Sigma^{+} \gamma$ and $\Xi^{* 0} \rightarrow \Xi^{0} \gamma$ are the same in quark model as shown in Table 15, we use the experimental value of the transition magnetic moments of $\Delta \rightarrow N \gamma$, $\Sigma^{*+} \rightarrow \Sigma^{+} \gamma, \Sigma^{* 0} \rightarrow \Lambda \gamma$ and $\mu_{\Xi^{* 0} \rightarrow \Xi^{0} \gamma}=\mu_{\Sigma^{*+} \rightarrow \Sigma^{+} \gamma}$, $\mu_{\Sigma^{*-} \rightarrow \Sigma^{-} \gamma}=\mu_{\Xi^{*-} \rightarrow \Xi^{-} \gamma}=(0 \pm 0.85) \mu_{N}$ to extract the six LECs: $\tilde{b}_{2}=4.22 \pm 0.53, \tilde{g}_{t 1}=-8.18 \pm 7.55, \tilde{g}_{t 2}=$ $-31.69 \pm 17.60, \tilde{d}_{1}=-0.96 \pm 0.72, \tilde{d}_{2}=-0.32 \pm 0.31$, $\tilde{d}_{3}=-1.62 \pm 0.34$. We list the numerical results up to $\mathcal{O}\left(p^{3}\right)$ in the fifth column in Table 4 . We also show the numerical results at each order in Fit B:

$$
\begin{aligned}
\mu_{\Delta^{+} \rightarrow p \gamma} & =-5.36 \times(1-0.41+0.07)=-3.54, \\
\mu_{\Delta^{0} \rightarrow n \gamma} & =-5.36 \times(1-0.41+0.07)=-3.54, \\
\mu_{\Sigma^{*+} \rightarrow \Sigma^{+} \gamma} & =5.36 \times(1-0.65+0.47)=4.43, \\
\mu_{\Sigma^{* 0} \rightarrow \Sigma^{0} \gamma} & =-2.68 \times(1-0.70+0.57)=-2.32, \\
\mu_{\Sigma^{* 0} \rightarrow \Lambda \gamma} & =4.64 \times(1-0.47+0.23)=3.54, \\
\mu_{\Sigma^{*-} \rightarrow \Sigma^{-} \gamma} & =0.32 \times(0+1-1.69)=-0.22, \\
\mu_{\Xi^{* 0} \rightarrow \Xi^{0} \gamma} & =5.36 \times(1-0.65+0.49)=4.51, \\
\mu_{\Xi^{*-} \rightarrow \Xi^{-} \gamma} & =0.32 \times(0+1-0.40)=0.19 .
\end{aligned}
$$

We have six LECs to fit, while we only have three accurate experimental inputs shown in Table 3. The data set is not rich enough to fix the LECs indeed. Although two schemes were introduced with the help of quark model, our fit is inevitably unstable. For the final uncertainties of the electromagnetic amplitudes considered, the uncertainties arise from the input $\operatorname{LECs}(D, F, \mathcal{C}, \mathcal{H}$, etc) account for about $10 \%$, while most arise from the experimental uncertainty. We hope more decuplet to octet baryon transition magnetic moments will be measured more precisely, thus the calculation may be more stable.

In Table 5, we compare our results obtained in the HBChPT with those from the other model calculations such as lattice QCD (LQCD) [70], chiral quark model (ChQM) [85], relativistic quark model (RQM) [86], effective mass quark model (EQM) [87], meson cloud (MS) [88], U-spin [89], QCD sum rules (QCD-SR) [67,90] and large $N_{c}[91,92]$. In some of the references, there are several fits. We list the fit whose value for the $\Delta \rightarrow N \gamma$ channel is closest to the empirical value. We also list the experimental values from the PDG [32].

We collect our numerical results of the decuplet to octet baryon transition electro quadrupole moments to the next-toleading order in Table 6. Up to $\mathcal{O}\left(p^{2}\right)$, we need include both the leading tree-level magnetic moments and the $\mathcal{O}\left(p^{1,2}\right)$ loop corrections. The $\mathcal{O}\left(p^{2}\right)$ loop corrections in Fig 2 are zero, so there is only one unknown low energy constant $\tilde{c}$ from Eq. (40). We use the experimental value of the $\Delta \rightarrow N \gamma$ transition magnetic dipole moment $G_{\mathrm{M} 1}=-3.12 \pm 0.14$ and the $\mathrm{E} 2$ to $\mathrm{M} 1$ ratio $R_{\mathrm{EM}}=(-2.5 \pm 0.5) \%$ [32] as input to extract the LEC $\tilde{c}=0.475 \pm 0.143$. The uncertainty in the tree value of Table 6 comes from that of $\tilde{c}$. We list the numer- 
Table 6 Electro quadrupole transition moments(in unit of 1)

\begin{tabular}{llccc}
\hline Process $\left(G_{\mathrm{E} 2}\right)$ & Tree value & Loop value & Total value & $R_{\mathrm{EM}}$ \\
\hline$\Delta^{+} \rightarrow p \gamma$ & $-0.548(165)$ & $0.473(164)$ & $-0.075(26)$ & $-2.5(9) \%$ \\
$\Delta^{0} \rightarrow n \gamma$ & $-0.548(165)$ & $0.473(164)$ & $-0.075(26)$ & $-2.5(9) \%$ \\
$\Sigma^{*+} \rightarrow \Sigma^{+} \gamma$ & $0.548(165)$ & $-0.502(147)$ & $0.046(13)$ & $-1.1(3) \%$ \\
$\Sigma^{* 0} \rightarrow \Sigma^{0} \gamma$ & $-0.274(83)$ & $0.255(72)$ & $-0.019(05)$ & $-0.9(3) \%$ \\
$\Sigma^{* 0} \rightarrow \Lambda \gamma$ & $0.475(143)$ & $-0.416(138)$ & $0.059(19)$ & $-1.8(6) \%$ \\
$\Sigma^{*-} \rightarrow \Sigma^{-} \gamma$ & 0 & $0.007(4)$ & $0.007(4)$ & $-0.7(2.1) \%$ \\
$\Xi^{* 0} \rightarrow \Xi^{0} \gamma$ & $0.548(165)$ & $-0.502(147)$ & $0.046(16)$ & $-3.9(2.2) \%$ \\
$\Xi^{*-} \rightarrow \Xi^{-} \gamma$ & 0 & $0.007(4)$ & $0.007(4)$ & \\
\hline
\end{tabular}

ical results of the transition electro quadrupole moments and the $\mathrm{E} 2$ to $\mathrm{M} 1$ ratio $R_{\mathrm{EM}}$ in Table 6.

We also calculate the M1 and E2 amplitudes and decay width of the decuplet to octet baryon transitions in the Appendix C. Both fits A and B lead to the same decay width for $\Sigma^{* 0} \rightarrow \Sigma^{0} \gamma$. The E2 amplitude of the $\Sigma^{* 0} \rightarrow \Sigma^{0} \gamma$ channel does not have any imaginary part because the $\pi^{+}$ and $\pi^{-}$loop contributions cancel each other as shown in Table 7. In other words, the pion loop contributions with the intermediate baryons $\Sigma^{*+}$ and $\Sigma^{*-}, \Sigma^{+}$and $\Sigma^{-}$cancel each other due to the exact $\mathrm{SU}(2)$ flavor symmetry. There are some imaginary parts in the loop results as shown in Eqs. (54), (56), (58), (60) and (63). Since the imaginary parts are much smaller than the real parts, we neglect the imaginary parts for magnetic moments, electric quadruple moments and decay width. The extracted M1 and E2 transition amplitudes and radiative decay widths may be useful for the future experimental measurement.

\section{Conclusions}

In short summary, we have systematically studied the decuplet to octet baryon transition magnetic moments up to the next-to-next-to-leading order in the framework of the heavy baryon chiral perturbation theory. With both the octet and decuplet baryon intermediate states in the chiral loops, we have systematically calculated the chiral corrections to the transition magnetic moments order by order. The chiral expansion converges rather well for the charged channels. We have also systematically calculated the electro quadrupole moments to the next-to-leading order and obtained the E2 to M1 ratio $R_{\mathrm{EM}}$ for decuplet to octet baryon transition, which suggests the d-wave component and deformed structure of the octet and decuplet baryons. Our results may be useful for future experimental measurement of the electro quadrupole multipole moments.
The decuplet to octet baryon transition magnetic moments of $\Delta^{+} \rightarrow p \gamma$ and $\Delta^{0} \rightarrow n \gamma$ are always the same because of the exact $\mathrm{SU}(2)$ spin-flavor symmetry. Comparing the $\mathcal{O}\left(p^{2}\right)$ and $\mathcal{O}\left(p^{3}\right) \Sigma^{*+} \rightarrow \Sigma^{+} \gamma$ transition magnetic moments to the experimental values, we want to emphasize the importance of the next-to-next-to-leading order chiral correction. As the current experimental data is not enough, we introduce two schemes to fit all LECs. Both fitting schemes lead to reasonably moderate convergence of the chiral expansion and agreement with the experimental data. However, when larger values of quark masses are introduced, such values cannot be trusted in extrapolations of lattice data. We hope that more decuplet to octet baryon transition magnetic moments like $\Xi^{* 0} \rightarrow \Xi^{0} \gamma$ will be measured more precisely in future experiments. Moreover, if considering the additional finitevolume corrections $[73,75,93,94]$, the analytical expressions derived in this work may be useful to the possible chiral extrapolation of the lattice simulations of the decuplet to octet baryon transition electromagnetic properties in the coming future.

Acknowledgements $\mathrm{H}$. S. Li is very grateful to $\mathrm{B}$. Zhou and L. Meng for very helpful discussions. This project is supported by the National Natural Science Foundation of China under Grants 11575008 , 11621131001,11705072 and 973 program. This work is also supported by the Double First-class University Construction Project of Northwest University and the Fundamental Research Funds for the Central Universities of Lanzhou University under Grants 223000-862637.

Data Availability Statement This manuscript has no associated data or the data will not be deposited. [Authors comment: The experimental data has already been shown in Table 5.]

Open Access This article is distributed under the terms of the Creative Commons Attribution 4.0 International License (http://creativecomm ons.org/licenses/by/4.0/), which permits unrestricted use, distribution, and reproduction in any medium, provided you give appropriate credit to the original author(s) and the source, provide a link to the Creative Commons license, and indicate if changes were made.

Funded by SCOAP 3 . 


\section{Integrals and loop functions}

We collect some common integrals and loop functions in this appendix.

6.1 Integrals with one or two meson propagators

$$
\begin{aligned}
\Delta & =i \int \frac{d^{d} l \lambda^{4-d}}{(2 \pi)^{d}} \frac{1}{l^{2}-m^{2}+i \epsilon} \\
& =2 m^{2}\left(L(\lambda)+\frac{1}{32 \pi^{2}} \ln \frac{m^{2}}{\lambda^{2}}\right), \\
L(\lambda) & =\frac{\lambda^{d-4}}{16 \pi^{2}}\left[\frac{1}{d-4}-\frac{1}{2}\left(\ln 4 \pi+1+\Gamma^{\prime}(1)\right)\right] .
\end{aligned}
$$

$$
I_{0}\left(q^{2}\right)=i \int \frac{d^{d} l \lambda^{4-d}}{(2 \pi)^{d}} \frac{1}{\left(l^{2}-m^{2}+i \epsilon\right)\left((l+q)^{2}-m^{2}+i \epsilon\right)}
$$

$$
= \begin{cases}-\frac{1}{16 \pi^{2}}\left(1-\ln \frac{m^{2}}{\lambda^{2}}-r \ln \left|\frac{1+r}{1-r}\right|\right)+2 L(\lambda) & \left(q^{2}<0\right) \\ -\frac{1}{16 \pi^{2}}\left(1-\ln \frac{m^{2}}{\lambda^{2}}-2 r \arctan \frac{1}{r}\right)+2 L(\lambda) & \left(0<q^{2}<4 m^{2}\right), \\ -\frac{1}{16 \pi^{2}}\left(1-\ln \frac{m^{2}}{\lambda^{2}}-r \ln \left|\frac{1+r}{1-r}\right|+i \pi r\right)+2 L(\lambda) & \left(q^{2}>4 m^{2}\right)\end{cases}
$$

$$
\begin{aligned}
& J_{1}(\omega)=-\omega J_{0}(\omega)+\Delta \\
& J_{2}(\omega)=\frac{1}{d-1}\left[\left(m^{2}-\omega^{2}\right) J_{0}(\omega)+\omega \Delta\right] \\
& J_{3}(\omega)=-\omega J_{1}(\omega)-J_{2}(\omega)
\end{aligned}
$$

6.3 Integrals with two baryon propagators and one meson propagator

$$
\begin{aligned}
& i \int \frac{d^{d} l \lambda^{4-d}}{(2 \pi)^{d}} \frac{\left[1, l_{\alpha}, l_{\alpha} l_{\beta}\right]}{\left(l^{2}-m^{2}+i \epsilon\right)(v \cdot l+i \epsilon)(\omega+v \cdot l+i \epsilon)} \\
& \quad=\left[\Gamma_{0}(\omega), v_{\alpha} \Gamma_{1}(\omega), g_{\alpha \beta} \Gamma_{2}(\omega)+v_{\alpha} v_{\beta} \Gamma_{3}(\omega)\right] \quad \omega \neq 0
\end{aligned}
$$

$\Gamma_{i}(\omega)=\frac{1}{\omega}\left[J_{i}(0)-J_{i}(\omega)\right]$ where $r=\sqrt{\left|1-4 m^{2} / q^{2}\right|}$.

6.2 Integrals with one baryon propagator and one meson propagator

$$
\begin{gathered}
i \int \frac{d^{d} l \lambda^{4-d}}{(2 \pi)^{d}} \frac{\left[1, l_{\alpha}, l_{\alpha} l_{\beta}\right]}{\left(l^{2}-m^{2}+i \epsilon\right)(\omega+v \cdot l+i \epsilon)}=\left[J_{0}(\omega), v_{\alpha} J_{1}(\omega), g_{\alpha \beta} J_{2}(\omega)+v_{\alpha} v_{\beta} J_{3}(\omega)\right], \\
J_{0}(\omega)= \begin{cases}\frac{-\omega}{8 \pi^{2}}\left(1-\ln \frac{m^{2}}{\lambda^{2}}\right)+\frac{\sqrt{\omega^{2}-m^{2}}}{4 \pi^{2}}\left(\operatorname{arccosh} \frac{\omega}{m}-i \pi\right)+4 \omega L(\lambda) & (\omega>m) \\
\frac{-\omega}{8 \pi^{2}}\left(1-\ln \frac{m^{2}}{\lambda^{2}}\right)+\frac{\sqrt{m^{2}-\omega^{2}}}{4 \pi^{2}} \arccos \frac{-\omega}{m}+4 \omega L(\lambda) & \left(\omega^{2}<m^{2}\right) \\
\frac{-\omega}{8 \pi^{2}}\left(1-\ln \frac{m^{2}}{\lambda^{2}}\right)-\frac{\sqrt{\omega^{2}-m^{2}}}{4 \pi^{2}} \operatorname{arccosh} \frac{-\omega}{m}+4 \omega L(\lambda) & (\omega<-m)\end{cases}
\end{gathered}
$$


6.4 Integrals with one baryon propagator and two meson propagators

$$
\begin{aligned}
& +J_{0}(\beta)\left(3 \beta \omega+\beta^{2} d^{2}-3 \beta d^{2} \omega+3 d^{2} \omega^{2}\right. \\
& \left.+\beta^{2} d-6(d-1)(v \cdot q)^{2}+(2 d-4) m^{2}-3 d \omega^{2}\right)
\end{aligned}
$$

$$
\begin{aligned}
i \int \frac{d^{d} l \lambda^{4-d}}{(2 \pi)^{d}} \frac{\left[1, l_{\alpha}, l_{\alpha} l_{\beta}, l_{v} l_{\alpha} l_{\beta}\right]}{\left(l^{2}-m^{2}+i \epsilon\right)\left((l+q)^{2}-m^{2}+i \epsilon\right)(\omega+v \cdot l+i \epsilon)}=\left[L_{0}(\omega), L_{\alpha}, L_{\alpha \beta}, L_{v \alpha \beta}\right], \beta=\omega-v \cdot q \\
L_{0}(\omega)= \begin{cases}\frac{1}{8 \pi^{2} v \cdot q}\left\{\frac{1}{2}\left[\left(\operatorname{arccosh} \frac{\beta}{m}\right)^{2}-\left(\operatorname{arccosh} \frac{\omega}{m}\right)^{2}\right]-i \pi \ln \frac{\sqrt{\beta^{2}-m^{2}}+\beta}{\sqrt{\omega^{2}-m^{2}}+\omega}\right\} & (\beta>m) \\
\frac{1}{16 \pi^{2} v \cdot q}\left[\left(\arccos \frac{-\omega}{m}\right)^{2}-\left(\arccos \frac{-\beta}{m}\right)^{2}\right] & \left(\beta^{2}<m^{2}\right) . \\
\frac{1}{16 \pi^{2} v \cdot q}\left[\left(\operatorname{arccosh} \frac{-\beta}{m}\right)^{2}-\left(\operatorname{arccosh} \frac{-\omega}{m}\right)^{2}\right] & (\beta-m)\end{cases}
\end{aligned}
$$

$$
\begin{aligned}
& L_{\alpha}=n_{1}^{\mathrm{I}} q_{\alpha}+n_{2}^{\mathrm{I}} v_{\alpha} \\
& L_{\alpha \beta}=n_{1}^{\mathrm{II}} g_{\alpha \beta}+n_{2}^{\mathrm{II}} q_{\alpha} q_{\beta}+n_{3}^{\mathrm{II}} v_{\alpha} v_{\beta}+n_{4}^{\mathrm{II}} v_{\alpha} q_{\beta}+n_{5}^{\mathrm{II}} q_{\alpha} v_{\beta} \\
& L_{\nu \alpha \beta}=n_{1}^{\mathrm{III}} q_{\nu} q_{\alpha} q_{\beta}+n_{2}^{\mathrm{III}} q_{\nu} q_{\alpha} v_{\beta} \\
& \quad+n_{3}^{\mathrm{II}} q_{\nu} q_{\beta} v_{\alpha}+n_{4}^{\mathrm{II}} q_{\alpha} q_{\beta} v_{v}+n_{5}^{\mathrm{II}} q_{\nu} g_{\alpha \beta} \\
& \quad+n_{6}^{\mathrm{III}} q_{\beta} g_{\nu \alpha}+n_{7}^{\mathrm{II}} q_{\alpha} g_{\nu \beta}+n_{8}^{\mathrm{II}} q_{\nu} v_{\alpha} v_{\beta} \\
& \quad+n_{9}^{\mathrm{II}} q_{\alpha} v_{v} v_{\beta}+n_{10}^{\mathrm{II}} q_{\beta} v_{\nu} v_{\alpha} \\
& \quad+n_{11}^{\mathrm{II}} g_{\nu \beta} v_{\alpha}+n_{12}^{\mathrm{II}} g_{\nu \alpha} v_{\beta}+n_{13}^{\mathrm{II}} g_{\alpha \beta} v_{v}+n_{14}^{\mathrm{II}} v_{\nu} v_{\alpha} v_{\beta}
\end{aligned}
$$

6.5 Explicit expressions of the scalar functions

$$
\begin{aligned}
n_{1}^{\mathrm{I}}= & \frac{2(v \cdot q) I_{0}-2(v \cdot q) L_{0} \omega+J_{0}(\beta)-J_{0}(\omega)}{2(v \cdot q)^{2}} \\
n_{2}^{\mathrm{I}}= & \frac{-J_{0}(\beta)+J_{0}(\omega)}{2(v \cdot q)} \\
n_{1}^{\mathrm{II}}= & \frac{L_{0} m^{2} 2(v \cdot q)+[2(v \cdot q)+\beta-2 \omega] J_{0}(\beta)+\omega J_{0}(\omega)}{(2(d-2))(v \cdot q)} \\
n_{2}^{\text {II }}= & \frac{1}{2(d-2)(v \cdot q)^{3}}[(v \cdot q) \\
& \left(2\left(L_{0}\left((d-2) \omega^{2}+m^{2}\right)-I_{0}(d-2) \omega\right)\right. \\
& \left.-I_{0}(d-2)(v \cdot q)\right) \\
& +J_{0}(\beta)(2(v \cdot q)+(d-1)(\beta-2 \omega)) \\
n_{3}^{\text {II }}= & \frac{-\omega J_{0}(\omega)+\beta J_{0}(\beta)}{2(v \cdot q)} \\
n_{4,5} \mathrm{II} & \frac{J_{0}(\beta)(\beta-\beta d+d \omega-2(v \cdot q))-2(v \cdot q) L_{0} m^{2}-\omega J_{0}(\omega)}{2(d-2)(v \cdot q)^{2}} \\
n_{1}^{\mathrm{II}}= & \frac{1}{2(d-2)(d-1)(v \cdot q)^{4}} \\
& {\left[-\beta d^{2} \Delta+d^{2} \Delta \omega+2 I_{3} d^{2}(v \cdot q)^{3}\right.} \\
& +I_{0} d^{2}(v \cdot q)^{2} \omega-2 d^{2}(v \cdot q) L_{0} \omega^{3}
\end{aligned}
$$

$$
\begin{aligned}
& +2 I_{0} d^{2}(v \cdot q) \omega^{2}-J_{0}(\omega) \\
& \left((2 d-4) m^{2}+\left(d^{2}-2 d+3\right) \omega^{2}\right) \\
& -\beta d \Delta+d \Delta \omega-6 I_{3} d(v \cdot q)^{3} \\
& -3 I_{0} d(v \cdot q)^{2} \omega-2 d \Delta(v \cdot q)-6 d(v \cdot q) L_{0} m^{2} \omega \\
& +6 d(v \cdot q) L_{0} \omega^{3}+2 I_{0} d(v \cdot q) m^{2}-6 I_{0} d(v \cdot q) \omega^{2} \\
& +4 I_{3}(v \cdot q)^{3}+2 I_{0}(v \cdot q)^{2} \omega-2 \Delta(v \cdot q) \\
& -6(v \cdot q) L_{0} m^{2} \omega-4(v \cdot q) L_{0} \omega^{3}-10 I_{0}(v \cdot q) m^{2} \\
& \left.+4 I_{0}(v \cdot q) \omega^{2}\right]
\end{aligned}
$$

$$
\begin{aligned}
& n_{2,3,4}^{\mathrm{II}}= \frac{1}{2(d-2)(d-1)(v \cdot q)^{3}}\left[-J_{0}(\beta)\right. \\
& {\left[4 \beta \omega+\beta^{2} d^{2}-2 \beta d^{2} \omega+d^{2} \omega^{2}\right.} \\
&+\beta^{2} d-2 \beta d \omega \\
&-4(d-1)(v \cdot q)^{2}+2 \beta(d-1)(v \cdot q)+(2 d-4) m^{2} \\
&\left.+d \omega^{2}-2 \omega^{2}\right]+\beta d^{2} \Delta-d^{2} \Delta \omega \\
&+2 J_{0}(\omega)\left(\omega^{2}+(d-2) m^{2}\right)+\beta d \Delta \\
&-d \Delta \omega+3 d \Delta(v \cdot q) \\
&+4 d(v \cdot q) L_{0} m^{2} \omega-2 I_{0} d(v \cdot q) m^{2} \\
&\left.-4(v \cdot q) L_{0} m^{2} \omega+8 I_{0}(v \cdot q) m^{2}\right] \\
& n_{5,6,7} \mathrm{II} \frac{1}{2(d-2)(d-1)(v \cdot q)^{2}}\left[J_{0}(\beta)\right. \\
&\left(3 \beta \omega+\beta^{2} d-3 \beta d \omega-2(d-1)(v \cdot q)^{2}\right. \\
&\left.+(d-2) m^{2}+2 d \omega^{2}-2 \omega^{2}\right) \\
&-\beta d \Delta+d \Delta \omega-2 d(v \cdot q) L_{0} m^{2} \omega \\
&+2 I_{0} d(v \cdot q) m^{2}-J_{0}(\omega)\left((d-2) m^{2}+\omega^{2}\right) \\
&\left.-2 \Delta(v \cdot q)+2(v \cdot q) L_{0} m^{2} \omega-6 I_{0}(v \cdot q) m^{2}\right] \\
& \frac{1}{2(d-2)(d-1)(v \cdot q)^{2}}\left[J_{0}(\beta)\right. \\
&\left(\beta\left(m^{2}-\omega^{2}\right)\right] \\
&\left.+(d-2) m^{2}\right) \\
&\left.n_{8,9,10}^{\mathrm{II}}=\left(d^{2}(\omega-\beta)-4(d-1)(v \cdot q)\right)-J_{0}(\omega)(d-2)(v \cdot q)-d \omega+2 \omega\right) \\
& \\
&
\end{aligned}
$$




$$
\begin{aligned}
n_{11,12,13}^{\mathrm{III}}= & \frac{1}{2(d-2)(d-1)(v \cdot q)}\left[-J_{0}(\beta)\right. \\
& \left(\beta(\beta d+2(d-1)(v \cdot q)-2 d \omega+2 \omega)+(d-2) m^{2}\right) \\
& \Delta(d(\beta-\omega)+2(d-1)(v \cdot q))+J_{0}(\omega)(d-2) \\
& \left.\left(m^{2}-\omega^{2}\right)\right] \\
n_{14}^{\mathrm{III}}= & \frac{1}{2(d-1)(v \cdot q)}\left[d \Delta(\beta-\omega)+J_{0}(\beta)\right. \\
& \left.\left(m^{2}-\beta^{2} d\right)+J_{0}(\omega)\left(d \omega^{2}-m^{2}\right)\right]
\end{aligned}
$$

\section{Coefficients of the loop corrections}

In this appendix, we collect the explicit formulae for the chiral expansion of the decuplet to octet baryon magnetic moments at $\mathcal{O}\left(p^{2}\right)$ in Table 7 and $\mathcal{O}\left(p^{3}\right)$ in Tables 8, 9, 10, 11 and 12 , respectively.

channel are zero. The reason is that the pion loop contributions from different intermediate states cancel each other. In other words, the pion loop contributions with the intermediate baryons $\Sigma^{*+}$ and $\Sigma^{*-}, \Sigma^{+}$ and $\Sigma^{-}$cancel each other due to the exact $\mathrm{SU}(2)$ flavor symmetry
Table 7 The coefficients of the loop corrections to the decuplet to octet baryon transition magnetic moments from Figs. 2(a) and 2(b). The sub-

\begin{tabular}{|c|c|c|c|c|c|c|}
\hline Process & $\gamma_{c}^{\pi}$ & $\gamma_{c}^{K}$ & $\gamma_{c}^{\eta}$ & $\gamma_{d}^{\pi}$ & $\gamma_{d}^{K}$ & $\gamma_{d}^{\eta}$ \\
\hline$\Delta^{+} \rightarrow p \gamma$ & $\frac{2}{\sqrt{3}}$ & $\frac{1}{\sqrt{3}}$ & 0 & $-\frac{4 \sqrt{3}}{3}\left(\tilde{g}_{t 1}+\tilde{g}_{t 2}\right)$ & $-\frac{2 \sqrt{3}}{3} \tilde{g}_{t 1}$ & 0 \\
\hline$\Delta^{0} \rightarrow n \gamma$ & $\frac{2}{\sqrt{3}}$ & $\frac{1}{\sqrt{3}}$ & 0 & $-\frac{4 \sqrt{3}}{3}\left(\tilde{g}_{t 1}+\tilde{g}_{t 2}\right)$ & $-\frac{2 \sqrt{3}}{3} \tilde{g}_{t 1}$ & 0 \\
\hline$\Sigma^{*+} \rightarrow \Sigma^{+} \gamma$ & $-\frac{1}{\sqrt{3}}$ & $-\frac{2}{\sqrt{3}}$ & 0 & $\frac{2 \sqrt{3}}{3} \tilde{g}_{t 1}$ & $\frac{4 \sqrt{3}}{3} \tilde{g}_{t 1}+\frac{4 \sqrt{3}}{3} \tilde{g}_{t 2}$ & 0 \\
\hline$\Sigma^{* 0} \rightarrow \Sigma^{0} \gamma$ & 0 & $\frac{\sqrt{3}}{2}$ & 0 & 0 & $-\sqrt{3} \tilde{g}_{t 1}-\frac{2 \sqrt{3}}{3} \tilde{g}_{t 2}$ & 0 \\
\hline$\Sigma^{* 0} \rightarrow \Lambda \gamma$ & -1 & $-\frac{1}{2}$ & 0 & $2 \tilde{g}_{t 1}+\frac{4}{3} \tilde{g}_{t 2}$ & $\tilde{g}_{t 1}+\frac{4}{3} \tilde{g}_{t 2}$ & 0 \\
\hline$\Sigma^{*-} \rightarrow \Sigma^{-} \gamma$ & $-\frac{1}{\sqrt{3}}$ & $\frac{1}{\sqrt{3}}$ & 0 & $\frac{2 \sqrt{3}}{3} \tilde{g}_{t 1}$ & $-\frac{2 \sqrt{3}}{3} \tilde{g}_{t 1}$ & 0 \\
\hline$\Xi^{* 0} \rightarrow \Xi^{0} \gamma$ & $-\frac{1}{\sqrt{3}}$ & $-\frac{2}{\sqrt{3}}$ & 0 & $\frac{2 \sqrt{3}}{3} \tilde{g}_{t 1}$ & $\frac{4 \sqrt{3}}{3} \tilde{g}_{t 1}+\frac{4 \sqrt{3}}{3} \tilde{g}_{t 2}$ & 0 \\
\hline$\Xi^{*-} \rightarrow \Xi^{-} \gamma$ & $-\frac{1}{\sqrt{3}}$ & $\frac{1}{\sqrt{3}}$ & 0 & $\frac{2 \sqrt{3}}{3} \tilde{g}_{t 1}$ & $-\frac{2 \sqrt{3}}{3} \tilde{g}_{t 1}$ & 0 \\
\hline
\end{tabular}
scripts " $\mathcal{T}$ " and " $\mathcal{N}$ " denote the decuplet and octet baryon within the loop while the superscripts denote the pseudoscalar meson. What calls for special attention is that the pion loop coefficients of $\Sigma^{* 0} \rightarrow \Sigma^{0} \gamma$

\begin{tabular}{lllll}
\hline Process & $\beta_{\mathcal{T}}^{\pi}$ & $\beta_{\mathcal{T}}^{K}$ & $\beta_{\mathcal{N}}^{\pi}$ & $\beta_{\mathcal{N}}^{K}$ \\
\hline$\Delta^{+} \rightarrow p \gamma$ & $\frac{10 \sqrt{3}}{9}$ & $\frac{2 \sqrt{3}}{9}$ & $-\frac{2 \sqrt{3}}{3}(D+F)$ & $-\frac{2 \sqrt{3}}{3}(D-F)$ \\
$\Delta^{0} \rightarrow n \gamma$ & $\frac{10 \sqrt{3}}{9}$ & $\frac{2 \sqrt{3}}{9}$ & $-\frac{2 \sqrt{3}}{3}(D+F)$ & $-\frac{2 \sqrt{3}}{3}(D-F)$ \\
$\Sigma^{*+} \rightarrow \Sigma^{+} \gamma$ & $-\frac{2 \sqrt{3}}{9}$ & $-\frac{10 \sqrt{3}}{9}$ & $\frac{2 \sqrt{3}}{3}(D-F)$ & $\frac{2 \sqrt{3}}{3}(D+F)$ \\
$\Sigma^{* 0} \rightarrow \Sigma^{0} \gamma$ & 0 & $\frac{2 \sqrt{3}}{3}$ & 0 & $-\frac{2 \sqrt{3}}{3} D$ \\
$\Sigma^{* 0} \rightarrow \Lambda \gamma$ & $-\frac{4}{3}$ & $-\frac{2}{3}$ & $\frac{4 D}{3}$ & $\frac{2}{3} D$ \\
$\Sigma^{*-} \rightarrow \Sigma^{-} \gamma$ & $-\frac{2 \sqrt{3}}{9}$ & $-\frac{10 \sqrt{3}}{9}$ & $\frac{2 \sqrt{3}}{3}(D-F)$ & $-\frac{2 \sqrt{3}}{3}(D-F)$ \\
$\Xi^{* 0} \rightarrow \Xi^{0} \gamma$ & $-\frac{2 \sqrt{3}}{9}$ & $\frac{2 \sqrt{3}}{9}$ & $\frac{2 \sqrt{3}}{3}(D-F)$ & $\frac{2 \sqrt{3}}{3}(D+F)$ \\
$\Xi^{*-} \rightarrow \Xi^{-} \gamma$ & $-\frac{2 \sqrt{3}}{9}$ & & & $-\frac{2 \sqrt{3}}{3}(D-F)$ \\
\hline
\end{tabular}

Table 8 The coefficients of the loop corrections to the decuplet to octet baryon transition magnetic moments from Figs. 2(c) and 2(d) 
Table 9 The coefficients of the loop corrections to the decuplet to octet baryon transition magnetic moments from Figs. 2(e) and 2(f)

\begin{tabular}{lllllll}
\hline Process & $\gamma_{a \mathcal{T}}^{\pi}$ & $\gamma_{a \mathcal{T}}^{K}$ & $\gamma_{a \mathcal{T}}^{\eta}$ & $\gamma_{a \mathcal{N}}^{\pi}$ & $\gamma_{a \mathcal{N}}^{K}$ & $\gamma_{a \mathcal{N}}^{\eta}$ \\
\hline$\Delta^{+} \rightarrow p \gamma$ & $\frac{10 \sqrt{3}}{9} b$ & $\frac{2 \sqrt{3}}{9} b$ & 0 & $-\frac{2}{\sqrt{3}}\left(b_{D}^{6}+b_{F}^{6}\right)(D+F)$ & $-\frac{2}{3 \sqrt{3}}\left[b_{D}^{6}(D+3 F)+3 b_{F}^{6}(D-F)\right]$ & 0 \\
$\Delta^{0} \rightarrow n \gamma$ & $\frac{10 \sqrt{3}}{9} b$ & $\frac{2 \sqrt{3}}{9} b$ & 0 & $-\frac{2}{\sqrt{3}}\left(b_{D}^{6}+b_{F}^{6}\right)(D+F)$ & $-\frac{2}{3 \sqrt{3}}\left[b_{D}^{6}(D+3 F)+3 b_{F}^{6}(D-F)\right]$ & 0 \\
$\Sigma^{*+} \rightarrow \Sigma^{+} \gamma$ & $\frac{2 \sqrt{3}}{9} b$ & $\frac{-14 \sqrt{3}}{9} b$ & 0 & $\frac{2}{3 \sqrt{3}}\left(2 b_{D}^{6}+3 b_{F}^{6}\right) F$ & $\frac{2}{3 \sqrt{3}}\left[b_{D}^{6}(3 D+F)+3 b_{F}^{6}(D-F)\right]$ & $\frac{2}{3 \sqrt{3}}\left(b_{D}^{6}+3 b_{F}^{6}\right) D$ \\
$\Sigma^{* 0} \rightarrow \Sigma^{0} \gamma$ & 0 & $\frac{2 \sqrt{3}}{3} b$ & 0 & $-\frac{2}{3 \sqrt{3}} b_{D}^{6}(D+2 F)$ & $-\frac{2}{3 \sqrt{3}}\left(b_{D}^{6} F+3 b_{F}^{6} D\right)$ & $-\frac{2}{3 \sqrt{3}} b_{D}^{6} D$ \\
$\Sigma^{* 0} \rightarrow \Lambda \gamma$ & $-\frac{4}{3} b$ & $-\frac{2}{3} b$ & 0 & $\frac{4}{3} b_{F}^{6} D$ & $2 b_{D}^{6} F+\frac{2}{3} b_{F}^{6} D$ & $\frac{2}{3} b_{D}^{6} D$ \\
$\Sigma^{*-} \rightarrow \Sigma^{-} \gamma$ & $\frac{2 \sqrt{3}}{9} b$ & $-\frac{2 \sqrt{3}}{9} b$ & 0 & $\frac{1}{3 \sqrt{3}}\left[6 b_{F}^{6} F-4 b_{D}^{6}(D+F)\right]$ & $\frac{1}{3 \sqrt{3}}\left[b_{D}^{6}(6 D-2 F)-6 b_{F}^{6}(D+F)\right]$ & $\frac{2}{\sqrt{3}}\left(-\frac{1}{3} b_{D}^{6}+b_{F}^{6}\right) D$ \\
$\Xi^{* 0} \rightarrow \Xi^{0} \gamma$ & $-\frac{\sqrt{6}}{9} b$ & $-\frac{10 \sqrt{3}}{9} b$ & 0 & $\frac{2}{\sqrt{3}} b_{F}^{6}(D-F)$ & $\frac{1}{\sqrt{3}}\left(b_{D}^{6}+2 b_{F}^{6}\right)(D+F)$ & $\frac{2}{3 \sqrt{3}} b_{D}^{6}(D+3 F)$ \\
$\Xi^{*-} \rightarrow \Xi^{-} \gamma$ & $\frac{\sqrt{3}}{9} b$ & $\frac{2 \sqrt{3}}{9} b$ & $-\frac{\sqrt{3}}{3} b$ & $-\frac{1}{\sqrt{3}}\left(b_{D}^{6}+b_{F}^{6}\right)(D-F)$ & $\frac{1}{3 \sqrt{3}}\left[-b_{D}^{6}(D+9 F)+6 b_{F}^{6}(D+F)\right]$ & $\frac{1}{3 \sqrt{3}}\left(b_{D}^{6}-3 b_{F}^{6}\right)(D+3 F)$ \\
\hline
\end{tabular}

Table 10 The coefficients of the loop corrections to the decuplet to octet baryon transition magnetic moments from Figs. 2(g) and 2(h)

\begin{tabular}{|c|c|c|c|c|c|c|}
\hline Process & $\gamma_{a \mathcal{T N}}^{\pi}$ & $\gamma_{a \mathcal{T} \mathcal{N}}^{K}$ & $\gamma_{a \mathcal{T} \mathcal{N}}^{\eta}$ & $\gamma_{a \mathcal{N} \mathcal{T}}^{\pi}$ & $\gamma_{a \mathcal{N} \mathcal{T}}^{K}$ & $\gamma_{a \mathcal{N} \mathcal{T}}^{\eta}$ \\
\hline$\Delta^{+} \rightarrow p \gamma$ & $\frac{10}{3 \sqrt{3}}(D+F)$ & $\frac{8}{3 \sqrt{3}} F$ & $-\frac{2}{3 \sqrt{3}}(D-3 F)$ & $\frac{4}{3 \sqrt{3}}$ & $\frac{2}{3 \sqrt{3}}$ & 0 \\
\hline$\Delta^{0} \rightarrow n \gamma$ & $\frac{10}{3 \sqrt{3}}(D+F)$ & $\frac{8}{3 \sqrt{3}} F$ & $-\frac{2}{3 \sqrt{3}}(D-3 F)$ & $\frac{4}{3 \sqrt{3}}$ & $\frac{2}{3 \sqrt{3}}$ & 0 \\
\hline$\Sigma^{*+} \rightarrow \Sigma^{+} \gamma$ & $-\frac{4}{3 \sqrt{3}}(D+3 F)$ & $-\frac{4}{3 \sqrt{3}}(D+3 F)$ & 0 & 0 & 0 & $-\frac{2}{\sqrt{3}}$ \\
\hline$\Sigma^{* 0} \rightarrow \Sigma^{0} \gamma$ & $\frac{8}{3 \sqrt{3}} F$ & $\frac{4}{3 \sqrt{3}}(D+F)$ & 0 & $\frac{2}{3 \sqrt{3}}$ & $-\frac{2}{3 \sqrt{3}}$ & $\frac{1}{\sqrt{3}}$ \\
\hline$\Sigma^{* 0} \rightarrow \Lambda \gamma$ & $-\frac{8}{9} D$ & $-\frac{4}{9}(D+9 F)$ & 0 & $-\frac{1}{3}$ & $-\frac{2}{3}$ & 0 \\
\hline$\Sigma^{*-} \rightarrow \Sigma^{-} \gamma$ & $-\frac{4}{3 \sqrt{3}}(D-F)$ & $\frac{4}{3 \sqrt{3}}(D-F)$ & 0 & $\frac{4}{3 \sqrt{3}}$ & $-\frac{4}{3 \sqrt{3}}$ & 0 \\
\hline$\Xi^{* 0} \rightarrow \Xi^{0} \gamma$ & $\frac{2}{3 \sqrt{3}}(D-F)$ & $-\frac{8}{3 \sqrt{3}}(D+2 F)$ & $\frac{2}{3 \sqrt{3}}(D+3 F)$ & $-\frac{2}{3 \sqrt{3}}$ & $\frac{2}{3 \sqrt{3}}$ & $-\frac{2}{\sqrt{3}}$ \\
\hline$\Xi^{*-} \rightarrow \Xi^{-} \gamma$ & $-\frac{4}{3 \sqrt{3}}(D-F)$ & $\frac{4}{3 \sqrt{3}}(D-F)$ & 0 & $\frac{4}{3 \sqrt{3}}$ & $-\frac{4}{3 \sqrt{3}}$ & 0 \\
\hline
\end{tabular}

Table 11 The coefficients of the loop corrections to the decuplet to octet baryon transition magnetic moments from Figs. 2(m) and 2(n)

\begin{tabular}{|c|c|c|c|c|c|c|}
\hline Baryons & $\gamma_{f \mathcal{T} 10}^{\pi}$ & $\gamma_{f \mathcal{T} 10}^{K}$ & $\gamma_{f \mathcal{T} 10}^{\eta}$ & $\gamma_{f \mathcal{T} 8}^{\pi}$ & $\gamma_{f \mathcal{T} 8}^{K}$ & $\gamma_{f \mathcal{T} 8}^{\eta}$ \\
\hline$\Delta^{+}$ & $\frac{5}{3}$ & $\frac{2}{3}$ & $\frac{1}{3}$ & 2 & 2 & 0 \\
\hline$\Delta^{0}$ & $\frac{5}{3}$ & $\frac{2}{3}$ & $\frac{1}{3}$ & 2 & 2 & 0 \\
\hline$\Delta^{-}$ & $\frac{5}{3}$ & $\frac{2}{3}$ & $\frac{1}{3}$ & 2 & 2 & 0 \\
\hline$\Sigma^{*+}$ & $\frac{8}{9}$ & $\frac{16}{9}$ & 0 & $\frac{5}{3}$ & $\frac{4}{3}$ & 1 \\
\hline$\Sigma^{* 0}$ & $\frac{8}{9}$ & $\frac{16}{9}$ & 0 & $\frac{5}{3}$ & $\frac{4}{3}$ & 1 \\
\hline$\Sigma^{*-}$ & $\frac{8}{9}$ & $\frac{16}{9}$ & 0 & $\frac{5}{3}$ & $\frac{4}{3}$ & 1 \\
\hline$\Xi^{* 0}$ & $\frac{1}{3}$ & 2 & $\frac{1}{3}$ & 1 & 2 & 1 \\
\hline$\Xi^{*-}$ & $\frac{1}{3}$ & 2 & $\frac{1}{3}$ & 1 & 2 & 1 \\
\hline
\end{tabular}


Table 12 The coefficients of the loop corrections to the decuplet to octet baryon transition magnetic moments from Figs. 2(o) and 2(p)

\begin{tabular}{lllllll}
\hline Baryons & $\gamma_{f \mathcal{N} 8}^{\pi}$ & $\gamma_{f \mathcal{N} 8}^{K}$ & $\gamma_{f \mathcal{N} 8}^{\eta}$ & $\gamma_{f \mathcal{N} 10}^{\pi}$ & $\gamma_{f \mathcal{N} 10}^{K}$ & $\gamma_{f \mathcal{N} 10}^{\eta}$ \\
\hline$p$ & $3(D+F)^{2}$ & $\frac{10}{3} D^{2}-4 D F+6 F^{2}$ & $\frac{1}{3}(D-3 F)^{2}$ & 4 & 1 & 0 \\
$n$ & $3(D+F)^{2}$ & $\frac{10}{3} D^{2}-4 D F+6 F^{2}$ & $\frac{1}{3}(D-3 F)^{2}$ & 4 & 1 & 0 \\
$\Sigma^{+}$ & $\frac{4}{3} D^{2}+8 F^{2}$ & $4\left(D^{2}+F^{2}\right)$ & $\frac{4}{3} D^{2}$ & $\frac{2}{3}$ & $\frac{10}{3}$ & 1 \\
$\Sigma^{0}$ & $\frac{4}{3} D^{2}+8 F^{2}$ & $4\left(D^{2}+F^{2}\right)$ & $\frac{4}{3} D^{2}$ & $\frac{2}{3}$ & $\frac{10}{3}$ & 1 \\
$\Lambda$ & $4 D^{2}$ & $\frac{4}{3}\left(D^{2}+9 F^{2}\right)$ & $\frac{4}{3} D^{2}$ & 3 & 2 & 0 \\
$\Sigma^{-}$ & $\frac{4}{3} D^{2}+8 F^{2}$ & $4\left(D^{2}+F^{2}\right)$ & $\frac{4}{3} D^{2}$ & $\frac{2}{3}$ & $\frac{10}{3}$ & 1 \\
$\Xi^{0}$ & $3(D-F)^{2}$ & $\frac{10}{3} D^{2}+4 D F+6 F^{2}$ & $\frac{1}{3}(D+3 F)^{2}$ & 1 & 3 & 1 \\
$\Xi^{-}$ & $3(D-F)^{2}$ & $\frac{10}{3} D^{2}+4 D F+6 F^{2}$ & $\frac{1}{3}(D+3 F)^{2}$ & 1 & 3 & 1 \\
\hline
\end{tabular}

\section{Appendix C: Transition amplitudes and decay width}

We collect the M1 and E2 amplitudes and decay width of the decuplet to octet baryon transitions in this appendix (Tables $13,14)$.

\section{Appendix D: Transition magnetic moments in quark model}

We collect the decuplet to octet baryon transition magnetic moments in quark model in this appendix. The transition moments are obtained by sandwiching Eq. (D1) between the decuplet and octet baryon states.

Table 13 M1 and E2 amplitudes and decay width of the decuplet to octet baryons in fit A

\begin{tabular}{llll}
\hline Process(fit A) & $f_{M 1} / \mathrm{GeV}^{-\frac{1}{2}}$ & $f_{E 2} / \mathrm{GeV}^{-\frac{1}{2}}$ & Decay width $\Gamma / \mathrm{MeV}$ \\
\hline$\Delta \rightarrow N \gamma$ & $-0.317+0.024 i$ & $0.008+0.018 i$ & 0.73 \\
$\Sigma^{*+} \rightarrow \Sigma^{+} \gamma$ & $0.246-0.022 i$ & $-0.003-0.002 i$ & 0.25 \\
$\Sigma^{* 0} \rightarrow \Sigma^{0} \gamma$ & $-0.129+0.010 i$ & 0.001 & 0.07 \\
$\Sigma^{* 0} \rightarrow \Lambda \gamma$ & $0.255+0.012 i$ & $-0.005-0.009 i$ & 0.43 \\
$\Sigma^{*-} \rightarrow \Sigma^{-} \gamma$ & $-0.012-0.002 i$ & $-0.001-0.002 i$ & $5.79 \times 10^{-4}$ \\
$\Xi^{* 0} \rightarrow \Xi^{0} \gamma$ & $0.282+0.023 i$ & $-0.003-0.002 i$ & 0.41 \\
$\Xi^{*-} \rightarrow \Xi^{-} \gamma$ & $0.010+0.002 i$ & $-0.001-0.002 i$ & $5.25 \times 10^{-4}$ \\
\hline
\end{tabular}

Table 14 M1 and E2 amplitudes and decay width of the decuplet to octet baryons in fit B. All the experimental values are from PDG [32]

\begin{tabular}{lllll}
\hline Process(fit B) & $f_{M 1} / \mathrm{GeV}^{-\frac{1}{2}}$ & $f_{E 2} / \mathrm{GeV}^{-\frac{1}{2}}$ & Decay width $\Gamma / \mathrm{MeV}$ & $\Gamma_{\exp } / \mathrm{MeV}$ \\
\hline$\Delta \rightarrow N \gamma$ & $-0.321+0.024 i$ & $0.008+0.018 i$ & 0.75 & 0.70 \\
$\Sigma^{*+} \rightarrow \Sigma^{+} \gamma$ & $0.244-0.022 i$ & $-0.003-0.002 i$ & 0.25 & 0.25 \\
$\Sigma^{* 0} \rightarrow \Sigma^{0} \gamma$ & $-0.128+0.010 i$ & 0.001 & 0.07 & - \\
$\Sigma^{* 0} \rightarrow \Lambda \gamma$ & $0.249+0.012 i$ & $-0.005-0.009 i$ & 0.42 & 0.45 \\
$\Sigma^{*-} \rightarrow \Sigma^{-} \gamma$ & $-0.012-0.002 i$ & $-0.001-0.002 i$ & $6.39 \times 10^{-4}$ & $<9.36 \times 10^{-3}$ \\
$\Xi^{* 0} \rightarrow \Xi^{0} \gamma$ & $0.236+0.023 i$ & $-0.003-0.002 i$ & 0.29 & $<0.38$ \\
$\Xi^{*-} \rightarrow \Xi^{-} \gamma$ & $0.010+0.002 i$ & $-0.001-0.002 i$ & $5.25 \times 10^{-4}$ & $<0.38$ \\
\hline
\end{tabular}


Table 15 Decuplet to octet baryon transition magnetic moments in quark model

\begin{tabular}{ll}
\hline Process & Expressions \\
\hline$\Delta \rightarrow N \gamma$ & $\frac{2 \sqrt{2}}{3}\left(\mu_{d}-\mu_{u}\right)$ \\
$\Sigma^{*+} \rightarrow \Sigma^{+} \gamma$ & $\frac{2 \sqrt{2}}{3}\left(\mu_{u}-\mu_{s}\right)$ \\
$\Sigma^{* 0} \rightarrow \Sigma^{0} \gamma$ & $\frac{\sqrt{2}}{3}\left(-\mu_{u}-\mu_{d}+2 \mu s\right)$ \\
$\Sigma^{* 0} \rightarrow \Lambda \gamma$ & $\frac{\sqrt{6}}{3}\left(\mu_{u}-\mu_{d}\right)$ \\
$\Sigma^{*-} \rightarrow \Sigma^{-} \gamma$ & $\frac{2 \sqrt{2}}{3}\left(\mu_{s}-\mu_{d}\right)$ \\
$\Xi^{* 0} \rightarrow \Xi^{0} \gamma$ & $\frac{2 \sqrt{2}}{3}\left(\mu_{u}-\mu_{s}\right)$ \\
$\Xi^{*-} \rightarrow \Xi^{-} \gamma$ & $\frac{2 \sqrt{2}}{3}\left(\mu_{d}-\mu_{s}\right)$ \\
\hline
\end{tabular}

$\vec{\mu}=\sum_{i} \mu_{i} \overrightarrow{\sigma^{i}}$

where

$\mu_{i}=\frac{e_{i}}{2 m_{i}}, i=u, d, s$.

The expressions for the decuplet to octet baryon transition magnetic moments are given in Table 15.

\section{References}

1. G.S. Adkins, C.R. Nappi, E. Witten, Nucl. Phys. B 228, 552 (1983)

2. T.D. Cohen, W. Broniowski, Phys. Rev. D 34, 3472 (1986)

3. D.B. Leinweber, R.M. Woloshyn, T. Draper, Phys. Rev. D 43, 1659 (1991)

4. D.B. Leinweber, T. Draper, R.M. Woloshyn, Phys. Rev. D 46, 3067 (1992)

5. E.E. Jenkins, M.E. Luke, A.V. Manohar, M.J. Savage, Phys. Lett. B 302, 482 (1993), [Erratum: Phys. Lett. B 388, 866 (1996)]

6. M.N. Butler, M.J. Savage, R.P. Springer, Phys. Rev. D 49, 3459 (1994)

7. M.K. Banerjee, J. Milana, Phys. Rev. D 54, 5804 (1996)

8. U.G. Meissner, S. Steininger, Nucl. Phys. B 499, 349 (1997)

9. S .L. Zhu, W .Y .P. Hwang, Z s Yang, Phys. Rev. D 57, 1527 (1998)

10. U.G. Meissner, Nucl. Phys. A 666, 51 (2000)

11. S.L. Zhu, S.J. Puglia, B.R. Holstein, M.J. Ramsey-Musolf, Phys. Rev. D 62, 033008 (2000)

12. S.J. Puglia, M.J. Ramsey-Musolf, Phys. Rev. D 62, 034010 (2000)

13. B. Kubis, U.G. Meissner, Eur. Phys. J. C 18, 747 (2001)

14. S.J. Puglia, M.J. Ramsey-Musolf, S.L. Zhu, Phys. Rev. D 63, 034014 (2001)

15. M.J. Savage, Nucl. Phys. A 700, 359 (2002)

16. D. Arndt, B.C. Tiburzi, Phys. Rev. D 68, 114503 (2003) [Erratum: Phys. Rev. D 69, 059904 (2004)]

17. I.C. Cloet, D.B. Leinweber, A.W. Thomas, Phys. Lett. B 563, 157 (2003)

18. M. Gockeler et al., [QCDSF Collaboration]. Phys. Rev. D 71, $034508(2005)$

19. V. Pascalutsa, M. Vanderhaeghen, Phys. Rev. Lett. 94, 102003 (2005)

20. C. Alexandrou, G. Koutsou, J.W. Negele, A. Tsapalis, Phys. Rev. D 74, 034508 (2006)
21. C. Hacker, N. Wies, J. Gegelia, S. Scherer, Eur. Phys. J. A 28, 5 (2006)

22. J. Arrington, C.D. Roberts, J.M. Zanotti, J. Phys. G 34, S23 (2007)

23. V. Pascalutsa, M. Vanderhaeghen, Phys. Rev. D 77, 014027 (2008)

24. H.W. Lin, K. Orginos, Phys. Rev. D 79, 074507 (2009)

25. C. Alexandrou et al., Phys. Rev. D 79, 014507 (2009)

26. C. Alexandrou, T. Korzec, G. Koutsou, C. Lorce, J.W. Negele, V. Pascalutsa, A. Tsapalis, M. Vanderhaeghen, Nucl. Phys. A 825, 115 (2009)

27. L .S. Geng, J. Martin Camalich, M .J. Vicente Vacas, Phys. Rev. D 80, 034027 (2009)

28. I.C. Cloet, W. Bentz, A.W. Thomas, Phys. Rev. C 90, 045202 (2014)

29. P.E. Shanahan et al., [CSSM and QCDSF/UKQCD Collaborations]. Phys. Rev. D 89, 074511 (2014)

30. M.E. Carrillo-Serrano, W. Bentz, I.C. Cloet, A.W. Thomas, Phys. Lett. B 759, 178 (2016)

31. V. Pascalutsa, M. Vanderhaeghen, S.N. Yang, Phys. Rep. 437, 125 (2007)

32. M. Tanabashi et al. [Particle Data Group], Phys. Rev. D 98(3), 030001 (2018)

33. R. Beck et al., Phys. Rev. Lett. 78, 606 (1997)

34. N. Sparveris et al., Eur. Phys. J. A 49, 136 (2013)

35. J.D. Bjorken, J.D. Walecka, Ann. Phys. 38, 35 (1966)

36. H.F. Jones, M.D. Scadron, Ann. Phys. 81, 1 (1973)

37. M.A.B. Beg, B.W. Lee, A. Pais, Phys. Rev. Lett. 13, 514 (1964)

38. C. Becchi, G. Morpurgo, Phys. Lett. 17, 352 (1965)

39. N. Isgur, G. Karl, R. Koniuk, Phys. Rev. D 25, 2394 (1982)

40. S.S. Gershtein, G.V. Jikia, Sov. J. Nucl. Phys. 34, 870 (1981) [Yad. Fiz. 34, 1566 (1981)]

41. M. Bourdeau, N.C. Mukhopadhyay, Phys. Rev. Lett. 58, 976 (1987)

42. S.A. Gogilidze, Yu. S. Surovtsev, F.G. Tkebuchava, Sov. J. Nucl. Phys. 45, 674 (1987) [Yad. Piz. 45, 1085 (1987)]

43. T.R. Hemmert, B.R. Holstein, N.C. Mukhopadhyay, Phys. Rev. D 51, 158 (1995)

44. A.J. Buchmann, Phys. Rev. Lett. 93, 212301 (2004)

45. L. Yu, X.L. Chen, W.Z. Deng, S.L. Zhu, Phys. Rev. D 73, 114001 (2006)

46. G. Ramalho, M.T. Pena, J. Phys. G 36, 115011 (2009)

47. A. Faessler, T. Gutsche, B.R. Holstein, V.E. Lyubovitskij, D. Nicmorus, K. Pumsa-ard, Phys. Rev. D 74, 074010 (2006)

48. S. Weinberg, Phys. A 96, 327 (1979)

49. J. Gasser, H. Leutwyler, Ann. Phys. 158, 142 (1984)

50. J. Gasser, H. Leutwyler, Nucl. Phys. B 250, 465 (1985)

51. V. Bernard, N. Kaiser, J. Kambor, U.G. Meissner, Nucl. Phys. B 388, 315 (1992)

52. E. Jenkins, A.V. Manohar, Phys. Lett. B 255, 558 (1991)

53. T. Hemmert, B.R. Holstein, J. Kambor, Phys. Lett. B 395, 89 (1997)

54. T. Hemmert, B.R. Holstein, J. Kambor, J. Phys. G 24, 1831 (1998)

55. M.N. Butler, M.J. Savage, R.P. Springer, Phys. Lett. B 304, 353 (1993)

56. G.C. Gellas, T.R. Hemmert, C.N. Ktorides, G.I. Poulis, Phys. Rev. D 60, 054022 (1999)

57. D. Arndt, B.C. Tiburzi, Phys. Rev. D 69, 014501 (2004)

58. T.A. Gail, T.R. Hemmert, Eur. Phys. J. A 28, 91 (2006)

59. V. Pascalutsa, M. Vanderhaeghen, Phys. Rev. Lett. 95, 232001 (2005)

60. G. Kaelbermann, J.M. Eisenberg, Phys. Rev. D 28, 71 (1983)

61. K. Bermuth, D. Drechsel, L. Tiator, J.B. Seaborn, Phys. Rev. D 37, 89 (1988)

62. D.H. Lu, A.W. Thomas, A.G. Williams, Phys. Rev. C 55, 3108 (1997)

63. A. Wirzba, W. Weise, Phys. Lett. B 188, 6 (1987)

64. A. Abada, H. Weigel, H. Reinhardt, Phys. Lett. B 366, 26 (1996)

65. H. Walliser, G. Holzwarth, Z. Phys. A 357, 317 (1997)

66. T.M. Aliev, A. Ozpineci, Nucl. Phys. B 732, 291 (2006)

67. L. Wang, F.X. Lee, AIP. Conf, Proc. 1182, 532 (2009) 
68. E. Jenkins, X d Ji, A .V. Manohar, Phys. Rev. Lett. 89, 242001 (2002)

69. A.J. Buchmann, J.A. Hester, R.F. Lebed, Phys. Rev. D 66, 056002 (2002)

70. D.B. Leinweber, T. Draper, R.M. Woloshyn, Phys. Rev. D 48, 2230 (1993)

71. C. Alexandrou et al., Phys. Rev. D 69, 114506 (2004)

72. C. Alexandrou, P. de Forcrand, H. Neff, J.W. Negele, W. Schroers, A. Tsapalis, Phys. Rev. Lett. 94, 021601 (2005)

73. N.H. Christ, C. Kim, T. Yamazaki, Phys. Rev. D 72, 114506 (2005)

74. G. Ramalho, M.T. Pena, Phys. Rev. D 80, 013008 (2009)

75. A. Agadjanov, V. Bernard, U.G. Meissner, A. Rusetsky, Nucl. Phys. B 886, 1199 (2014)

76. R.A. Briceno, M.T. Hansen, Phys. Rev. D 92(7), 074509 (2015)

77. M.E. Luke, A.V. Manohar, Phys. Lett. B 286, 348 (1992)

78. R. Davidson, N.C. Mukhopadhyay, R. Wittman, Phys. Rev. Lett. 56, 804 (1986)

79. S. Scherer, Adv. Nucl. Phys. 27, 277 (2003)

80. V. Bernard, N. Kaiser, U.G. Meissner, Int. J. Mod. Phys. E 4, 193 (1995)
81. W. Rarita, J. Schwinger, Phys. Rev. 60, 61 (1941)

82. M.N. Butler, M.J. Savage, R.P. Springer, Nucl. Phys. B 399, 69 (1993)

83. H.S. Li, Z.W. Liu, X.L. Chen, W.Z. Deng, S.L. Zhu, Phys. Rev. D 95(7), 076001 (2017)

84. G. Ecker, Prog. Part. Nucl. Phys. 35, 1 (1995)

85. H.C. Kim, M. Polyakov, M. Praszalowicz, G.S. Yang, K. Goeke, Phys. Rev. D 71, 094023 (2005)

86. G. Ramalho, K. Tsushima, Phys. Rev. D 87(9), 093011 (2013)

87. R. Dhir, R.C. Verma, Eur. Phys. J. A 42, 243 (2009)

88. G. Ramalho, K. Tsushima, Phys. Rev. D 88, 053002 (2013)

89. D. Keller, K. Hicks, Eur. Phys. J. A 49, 53 (2013)

90. S.T. Hong, Phys. Rev. D 76, 094029 (2007)

91. E.E. Jenkins, Phys. Rev. D 85, 065007 (2012)

92. R.F. Lebed, D.R. Martin, Phys. Rev. D 70, 016008 (2004)

93. L. Lellouch, M. Luscher, Commun. Math. Phys. 219, 31 (2001)

94. C.J.D. Lin, G. Martinelli, C.T. Sachrajda, M. Testa, Nucl. Phys. B 619, 467 (2001) 